\title{
Generation of galactic disc warps due to intergalactic accretion flows onto the disc
}

\author{
M. López-Corredoira ${ }^{1,2}$, J. Betancort-Rijo ${ }^{2,3}$, and J. E. Beckman ${ }^{2,4}$ \\ 1 Astronomisches Institut der Universität Basel, Venusstrasse 7, 4102-Binningen, Switzerland \\ 2 Instituto de Astrofísica de Canarias, 38200 La Laguna, Tenerife, Spain \\ 3 Departamento de Astrofísica, Universidad de La Laguna, Tenerife, Spain \\ 4 Consejo Superior de Investigaciones Científicas (CSIC), Spain
}

Received 29 March 2001 / Accepted 5 February 2002

\begin{abstract}
A new method is developed to calculate the amplitude of the galactic warps generated by a torque due to external forces. This takes into account that the warp is produced as a reorientation of the different rings which constitute the disc in order to compensate the differential precession generated by the external force, yielding a uniform asymptotic precession for all rings.

Application of this method to gravitational tidal forces in the Milky Way due to the Magellanic Clouds leads to a very low amplitude of the warp, as has been inferred in previous studies; so, tidal forces are unlikely to generate warps, at least in the Milky Way. If the force were due to an extragalactic magnetic field, its intensity would have to be very high, greater than $1 \mu \mathrm{G}$, to generate the observed warps.

An alternative hypothesis is explored: the accretion of the intergalactic medium over the disk. A cup-shaped distortion is expected, due to the transmission of the linear momentum; but, this effect is small and the predominant effect turns out to be the transmission of angular momentum, i.e. a torque giving an integral-sign shape warp. The torque produced by a flow of velocity $\sim 100 \mathrm{~km} \mathrm{~s}^{-1}$ and baryon density $\sim 10^{-25} \mathrm{~kg} / \mathrm{m}^{3}$ is enough to generate the observed warps and this mechanism offers quite a plausible explanation. First, because this order of accretion rate is inferred from other processes observed in the Galaxy, notably its chemical evolution. The inferred rate of infall of matter, $\sim 1 M_{\odot} / \mathrm{yr}$, to the Galactic disc that this theory predicts agrees with the quantitative predictions of this chemical evolution resolving key issues, notably the G-dwarf problem. Second, the required density of the intergalactic medium is within the range of values compatible with observation. By this mechanism, we can explain the warp phenomenon in terms of intergalactic accretion flows onto the disk of the galaxy.
\end{abstract}

Key words. galaxies: structure - galaxy: structure - galaxies: interactions - galaxies: kinematic and dynamics galaxies: magnetic fields

\section{Introduction}

Many spiral galaxies present warps, distortions to a flat disc with an integral-sign shape. The Milky Way is an example (Burton 1988, 1992). Indeed, most of the spiral galaxies for which we have relevant information on their structure (because they are edge on and they are nearby) present warps. Sánchez-Saavedra et al. (1990) and Reshetnikov \& Combes (1998) show that nearly half of the spiral galaxies of selected samples are warped, and many of the rest might also be warped since warps in galaxies with low inclination are difficult to detect.

The intergalactic magnetic field has been suggested as the cause of galactic warps (Battaner et al. 1990; Battaner et al. 1991; Battaner \& Jiménez-Vicente 1998). This is

Send offprint requests to: M. López-Corredoira, e-mail: martinlc@astro.unibas.ch in our opinion a serious proposal (an opinion not held, however, by Binney 2000) which could explain many of the observations, although observational support is still controversial. The postulated alignment of warps of different galaxies (Battaner et al. 1991) and the differences between the gaseous and stellar warps (Porcel et al. 1997) can have alternative explanations, as we shall see in the present paper.

Gravitational tidal effects on the Milky Way due to the Magellanic Clouds are not enough to justify the observed amplitude of the warp. Hunter \& Toomre (1969) calculated that the Clouds with mass $M_{\text {sat }}=10^{10} M_{\odot}$ and distance $d=55 \mathrm{kpc}$ would generate a warp of amplitude less than $117 \mathrm{pc}$ at radius of $16 \mathrm{kpc}$ in the most favourable case instead of the observed 2 or $3 \mathrm{kpc}$. The Magellanic Clouds are near the pericentres of their orbits around Milky Way (Murai \& Fujimoto 1980; Lin \& Lynden-Bell 1982), so it 
is not expected that this amplitude could be greater due to a closer approach of the Magellanic Clouds in a recent past.

Weinberg (1998) proposed a mechanism to amplify the tidal effects due to a satellite by means of an intermediate massive halo around the galactic disc, but García-Ruiz et al. (2000) have found that the orientation of the warp is not compatible with the generation of warps by means of this mechanism if the satellites are the Magellanic Clouds. Quantitatively, a better prospect would be the Sagittarius dwarf galaxy (Ibata \& Razoumov 1998) since tidal effects are proportional to $\frac{M_{\mathrm{sat}}}{d_{\mathrm{sat}}^{3}}$, the galactocentric distance to this dwarf Galaxy is only $16 \mathrm{kpc}$ and its mass $\sim 10^{9} M_{\odot}$ (see Sect. 2.2).

Binney's (1992) review concludes that halos dominate the dynamics of the warps, although he also points out that "warps will in the end prove to be valuable probes of cosmic infall and galaxy formation". In a subsequent paper (Jiang \& Binney 1999), cosmic infall is used to explain the reorientation of a massive Galactic halo (8 degrees per Gyr) which produces a warp in the disc. This model requires a halo ten times more massive than the disc, an extremely high accretion rate (3 disc masses in 0.9 Gyr) and, in this scenario, after a sufficiently long time, the angular momentum of the Galaxy would become parallel to the direction of the falling matter causing the warp to decay. This last problem might be solved by including a prolate halo (Ideta et al. 2000). The general case of warps produced by the dynamical friction between a misaligned rotating halo and disk was also studied by Debattista \& Sellwood (1999). Other proposals which include a massive halo have also serious flaws or not very plausible assumptions (Nelson \& Tremaine 1995; Binney et al. 1998).

In spite of the importance which is given to the halo in the dynamics of galaxies, it is quite possible that they may not even play a major role in the formation of warps. The mass of the halo of the Galaxy is not especially well determined and the mass fraction of galaxy halos might be small (see, for instance: Nelson 1988; Battaner et al. 1992; Evans 2001 and references therein). We should emphasize here that the presence or absence of a very massive halo will not modify qualitatively the arguments presented below and would imply quantitative changes within the same order of magnitude. We will argue in this paper that warps can be generated without massive halos or magnetic fields, although our results are perfectly compatible with the existence of these.

We aim here an alternative solution to those hitherto hypothesized. The mechanism of generation of warps which is explained in this paper solves these previous difficulties and does not require implausible assumptions. It is even simpler than the hypotheses previously proposed; it requires only the infall of a very low density intergalactic medium onto the disc without the dynamical intervention of an intermediate halo. It is a very simple idea but it works well, as will be shown below.
An analytical calculation is performed to reduce the problem to a differential equation and some integrals, which are subsequently solved by means of numerical algorithms. A new method is developed in Sect. 2 to calculate the warp parameters from an induced external torque which takes into account the interaction between all rings of the galactic disc. The external torque induced by the accretion is calculated in Sect. 3, which allows us to estimate the required density of the inflow, in Sect. 4.

\section{Warp induced by an external torque}

In this section, we will explain the mechanism of generation of warps in a galactic disc due to a net external torque. This general method is applicable to any kind of torque acting over the disc and will be used to derive the properties of the warp induced by an intergalactic accretion flow.

In order to describe a warp in a galactic disc, we will use the usual model of tilted rings (Rogstad et al. 1974): the disc is taken to be a set of concentric rings, each of radius between $R_{i}$ and $R_{i}+\mathrm{d} R$ having angle inclination $\alpha_{i}$ with respect to the central disc and intersecting its plane at two nodes, perpendicular to the points on the ring where the elevation is maximum and minimum. The line joining the nodes ("line of nodes"), will be taken as common to all the rings, i.e., the nodes for all rings are aligned, as observed in our Galaxy; this is the equilibrium state in our model. Therefore, the only parameters which define the warp are: the direction of the line of nodes and a function, $\alpha(R)$, the maximum angular elevation of the ring of radius $R$ with respect to the plane defined by the central disc.

A torque applied to a rotating rigid body (in our case, the rigid body is a ring) produces a precession in it, as in the case of the equinox precession of the Earth. The importance of precession in galaxies was indeed first recognized by Lynden-Bell (1965). This section describes how the differential precession in successive rings generates a warp due to their redistribution. The general formalism included here is applicable to any kind of torque induced by a external force over a set of nearly coplanar rings. In the following section, the calculation of the torque for the specific case of the intergalactic accretion flows will be derived.

Other analytical approaches to the problem were used previously. For instance, the consideration of the warp as a product of equilibrium forces of vertical components, such that $F_{\text {ext }}, z=F_{\text {grav }}, z$, where $F_{\text {grav }}$ is the gravitational force due to an axisymmetric potential (used in Kahn \& Woltjer 1959; Binney 1991; Binney 1992 (his Sect. 2: "Naive theory"); Battaner \& Jiménez-Vicente 1998). Since the orbit within a ring is tilted, the centrifugal forces will cancel both radial and vertical components of the gravitational forces so this consideration is not valid. Our approach has some advantages and finer calculations than these papers. A mass in the centre of the galaxy cannot provide any torque to the rings. However, the 
axisymmetric potential of the disc is distorted by the warp (Binney 1992), and these non-axisymmetries are those responsible for the warp itself. The differential orientation of the successive rings rather than a point mass placed in the centre produces a torque. The approach used in Hunter \& Toomre (1969) is much better although the analysis is very different to the one presented here.

\subsection{Equations}

We consider the disc to be made of material on circular orbits with angular velocity $\omega_{\text {rot }}(R)$. If initially all the orbits are in the same plane (the plane of the disc), perpendicular to a unit vector $\boldsymbol{k}$, under the influence of an external torque with a non-vanishing component perpendicular to $\boldsymbol{k}$ the orbits will precess. If the precession is not equal for all orbits they will not remain in the same plane, so that the vector perpendicular to the plane of a given orbit $\boldsymbol{k}(R, t)$ will be a function of $R$ and of time $t$.

In the general case, the component of the external torque along the vector $\boldsymbol{k}(R, t)$ will induce changes in $\omega_{\text {rot }}(R)$. In addition, other moments of the external force may produce changes in the shape of the orbits. However in the applications relevant to the present problem these changes are negligible and we will take the orbits to remain circular, and with essentially constant $\omega_{\text {rot }}(R)$. In this case the dynamics of the disc under an external torque may be reduced to an equation in partial derivatives for $\boldsymbol{k}(R, t)$, or in other words a set of an infinite number of ordinary differential equations, (one for each value of $R$ ). The unit vector $\boldsymbol{k}(R, t)$ is defined by two parameters which are in fact angles.

The equations for the evolving system, (throughout this work we use equations valid for an inertial, nonrotating frame, and at no stage do we use a rotating frame) averaged over times longer than the orbital periods are:

$\frac{\mathrm{d} \boldsymbol{J}}{\mathrm{d} t}(R, t)=\boldsymbol{\tau}(R, t)=\boldsymbol{\tau}_{\text {ext }}(R, t)+\boldsymbol{\tau}_{\text {int }}(R, t)$,

where $\boldsymbol{J}(R, t)$ is the angular momentum for the ring with radius between $R$ and $R+\mathrm{d} R$, and $\tau_{\text {ext }}, \boldsymbol{\tau}_{\text {int }}$ represent the average torque in the ring due to the external force, and to the gravitational interaction with the rest of the disc respectively.

\subsubsection{External torque}

The external torque is:

$\boldsymbol{\tau}_{\text {ext }}(R, t)=\tau_{\text {ext }}[\boldsymbol{k}(R, t) . \boldsymbol{u}, R] \boldsymbol{i}_{0}$

$\boldsymbol{i}_{0} \equiv \boldsymbol{j}_{0} \times \boldsymbol{k}_{0}$,

where $\boldsymbol{u}$ is a unit vector in the direction associated with the cause of the external torque, which may be e.g., the direction of the infalling gas flow or the position of a perturbing galaxy, $\boldsymbol{j}_{0}$ is a unit vector parallel to the projection of $\boldsymbol{u}$ onto the initial plane (perpendicular to $\boldsymbol{k}_{0}$ ), $\tau_{\text {ext }}(x, R)$ is a function of the cosine of the angle between $\boldsymbol{u}$ and $\boldsymbol{k}(R, t)$, which characterizes the specific mechanism giving rise to the torque.

\subsubsection{Internal torque}

The internal torque is:

$\tau_{\text {int }}(R, t)=\int_{\text {all rings }}\left[\mathrm{d} \tau_{S}(R, t) \mathrm{d} R\right] \mathrm{d} S$,

and $\mathrm{d} \tau_{S}(R, t) \mathrm{d} R \mathrm{~d} S$ is the gravitational torque that the ring of radius between $S$ and $S+\mathrm{d} S$ produces on the ring of radius between $R$ and $R+\mathrm{d} R$, which is:

$\mathrm{d} \boldsymbol{\tau}_{S}(R, t) \mathrm{d} R \mathrm{~d} S=G \sigma(R) \sigma(S) \mathrm{d} R \mathrm{~d} S$

$\times \tau_{\text {int }}(\boldsymbol{k}(R, t) \cdot \boldsymbol{k}(S, t), R, S) \boldsymbol{k}(R, t) \times \boldsymbol{k}(S, t)$,

where $\tau_{\text {int }}\left(\cos \alpha_{R, S}, R, S\right)$ is a well determined function (the same for any mechanism) of the cosine of the angle $\alpha_{R, S}$ between the planes of the two orbits. Explicitly:

$\mathrm{d} \tau_{S}(R, t) \mathrm{d} R \mathrm{~d} S=G \sigma(R) \sigma(S) \mathrm{d} R \mathrm{~d} S \frac{S^{2}}{R} \int_{0}^{2 \pi} \mathrm{d} \phi_{1} \int_{0}^{2 \pi} \mathrm{d} \phi_{2}$

$\times \overline{\left[1+(S / R)^{2}-2(S / R)\left(\sin \phi_{1} \sin \phi_{2}+\cos \phi_{1} \cos \phi_{2} \cos \alpha_{R, S}\right)\right]^{3 / 2}}$

$\times\left[\sin \phi_{1} \sin \alpha_{R, S} \cos \phi_{2} \boldsymbol{i}(R, S, t)-\cos \phi_{1} \sin \alpha_{R, S} \cos \phi_{2} \boldsymbol{j}(R, S, t)\right.$

$\left.+\left[\cos \phi_{1} \sin \phi_{2}-\cos \phi_{2} \sin \phi_{1} \cos \alpha_{R, S}\right] \boldsymbol{k}(R, t)\right]$,

$\alpha_{R, S} \equiv \alpha(S, t)-\alpha(R, t) ; \quad \alpha(0, t) \equiv 0 ;$

$\cos \alpha_{R, S} \equiv \boldsymbol{k}(R, t) \cdot \boldsymbol{k}(S, t)$

$\boldsymbol{i}(R, S, t) \equiv \boldsymbol{j}(R, S, t) \times \boldsymbol{k}(R, t) ; \quad \boldsymbol{j}(R, S, t) \equiv \frac{\boldsymbol{k}(R, t) \times \boldsymbol{k}(S, t)}{|\boldsymbol{k}(R, t) \times \boldsymbol{k}(S, t)|}$.

The torque between two rings, $\mathrm{d} \tau_{S}(R, t) \mathrm{d} R \mathrm{~d} S$, is proportional to $-\boldsymbol{j}(R, S, t)$, and the external torque must be parallel to this. The $z$-component of the external torque, if this were present, would produce an acceleration in the rotation of the disc rather than warping.

Other components of a galaxy produce negligible contribution. The bulge in practice contribute negligibly to the torque: firstly, because it is more spherical than the rings and a spherical distribution of mass produces no torque; and, secondly, because the distance of the bulge to the outer rings is large enough for these to produce negligible effects (the torque is proportional to $S^{2} / R$ for small $S$, where $S$ is the radius of the structure). Numerical experiments were carried out which confirmed this point. A massive halo, if it exists, would produce an extra internal torque if the halo is non spherical. In practice, a massive non-spherical halo will change quantitatively the amplitude of the warp but, qualitatively the mechanism will be the same; the warp amplitude will be reduced, never increased, because a massive halo would keep the rings more tightly bound. At present, no precise calculations including non-spherical massive halos are given here, but the net result would be to increase the required accretion rates inferred below. Only the inner mass of the 
halo ellipsoid with semimajor axis equal to $R$ produce net torque, assuming there is a constant ellipticity halo, whose quadrupole is $\sim \frac{4}{15} \frac{v_{\mathrm{rot}}^{2} \epsilon R^{3} f_{h}(R)}{G^{2}}\left(v_{\text {rot }}(R)\right.$ is the rotation velocity of the Galaxy; $\epsilon$ is the eccentricity of the ellipsoids; a mass distribution for the halo derived from a hypothetical flat rotation curves due to a dark halo is assumed; $f_{\mathrm{h}}(R)$ fraction of the mass $M(R)$ embedded in the halo) while the disc quadrupole component is $\sim 12 \pi \sigma\left(R_{0}\right) \mathrm{e}^{R_{0} / h_{R}} h_{R}^{4}$ for a model such as (15). The disc dominates at $R<22 \mathrm{kpc}$ for $\epsilon=0.2$ and $f_{h}=0.5$ (Kuijken \& Dubinski 1995), and the contribution of the halo for $R<16 \mathrm{Kpc}$ is less than $40 \%$ of the disc contribution. This means that the order of magnitude of the torque will be not affected by the inclusion of the massive halo, and will be perhaps affected by a factor less than $\sim 1.4$ at $R<16 \mathrm{kpc}$.

For small angles, in a linear approximation, the proportionality which results is:

$$
\lim _{\alpha_{R, S} \longrightarrow 0} \mathrm{~d} \tau_{S}(R) \mathrm{d} R \mathrm{~d} S \propto-\alpha_{R, S} \boldsymbol{j}(R, S, t) .
$$

\subsubsection{Dynamics and evolution of the warp}

The precession velocity of $\boldsymbol{k}(R, t)$ is much less than $\omega_{\text {rot }}(R, t)$, so we have

$$
\boldsymbol{J}(R, t) \approx I \omega_{\mathrm{rot}}(R, t) \boldsymbol{k}(R, t)
$$

$I=2 \pi R^{3} \sigma(R) \mathrm{d} R$,

where $I$ is the moment of inertia of the ring, and $\sigma(R)$ the surface density of the disc.

$\sigma(R)=\int_{-\infty}^{\infty} \mathrm{d} z \rho_{\text {disc }}(R, z)$,

where $\rho_{\text {disc }}$ is its spatial density, independent of $\phi$ in an assumed axisymmetric case.

This approximation is typical in planetary precession, for instance. Using this expression in (1), and calling $\boldsymbol{\tau}_{\|}$, $\boldsymbol{\tau}_{\perp}$ the components of $\boldsymbol{\tau}$ along $\boldsymbol{k}(R, t)$ and perpendicular to $\boldsymbol{k}(R, t)$ respectively, we obtain

$\left|I \dot{\omega}_{\text {rot }}(R, t)\right|=\left|\boldsymbol{\tau}_{\|}\right|=|\boldsymbol{k}(R, t) . \boldsymbol{\tau}(R, t)| ;$

$\frac{\mathrm{d} \boldsymbol{k}(R, t)}{\mathrm{d} t}(R, t)=\frac{\boldsymbol{\tau}_{\perp}}{I \omega_{\mathrm{rot}}(R, t)}$

$=\frac{[\boldsymbol{\tau}(R, t)-(\boldsymbol{k}(R, t) \cdot \boldsymbol{\tau}(R, t)) \boldsymbol{k}(R, t)]}{I \omega_{\mathrm{rot}}(R, t)}$.

With the set of initial conditions $\boldsymbol{k}\left(R, t_{0}\right)=\boldsymbol{k}_{0} \equiv$ $(0,0,1) \forall R$, Eq. (10) can be integrated to give the configuration of the orbits, and hence the shape of the disc, at any time.

The method described here is not restricted to linear perturbations but is valid as long as the deformed disc is describable by a set of tilted rings. However, for large deformations the orbit cannot remain flat and the approximation will fail. This is not the case however in the examples given in the present discussion. It must be noted that Eq. (10) for $\left(\frac{d \boldsymbol{k}}{\mathrm{d} t}\right)$ is a first order differential equation, which implies that it is the angular velocities and not their time derivative that are determined by the torque. So no matter how large (modulus of vector $\frac{\mathrm{d} \boldsymbol{k}}{\mathrm{d} t}$ ) may be, if the torque vanishes instantly so will $\frac{\mathrm{d} k}{\mathrm{~d} t}$. This is due to the fact that the energy associated with $\frac{\mathrm{d} \boldsymbol{k}}{\mathrm{d} t}$ is much smaller than the energy corresponding to $\omega_{\text {rot }}$; the full equations are obviously second order.

\subsubsection{Equilibrium configuration}

Here we are interested in the stationary, or equilibrium configuration that we assume exists, rather than in the evolution. It is clear that in this situation all orbits (or rings) must precess around $\boldsymbol{u}$ at the same constant speed, $\omega_{p}$, keeping $\boldsymbol{k}(R, t) \cdot \boldsymbol{u}$ constant in time, though different for different orbits. For $\alpha(R)$ and the precession velocity, $\omega_{p}(R)$, to be independent of time, $\boldsymbol{\tau}(R, t)$ must be perpendicular to $\boldsymbol{k}(R, t)$ and $\boldsymbol{u}$ for any $R$. Since this holds for $\boldsymbol{\tau}_{\text {ext }}(R, t)$ it must also hold for $\boldsymbol{\tau}_{\text {int }}(R, t)$. However, examining the expression for this last vector in (10), for this condition to hold for all orbits, they must all intersect along the same straight line, parallel to the unit vector $\boldsymbol{i}$. In this case the position of the orbits is simply given by the function $\alpha(R) \equiv \cos ^{-1}\left(\boldsymbol{k}(R, t) \cdot \boldsymbol{k}_{0}\right)$.

If the vector $\boldsymbol{k}(R, t)$ precesses around $\boldsymbol{u}$ with angular velocity $\omega_{p}(R)$ we then have:

$\omega_{p}(R) \boldsymbol{u} \times \boldsymbol{k}(R, t)=\frac{\mathrm{d} \boldsymbol{k}}{\mathrm{d} t}$.

From this equation we obtain $\omega_{p}(R)[\alpha(R)]$ as a function of $\alpha(R)$. Now, since one of the conditions for a stationary configuration is that $\omega_{p}(R)$ be independent of $R$, setting the derivative of this function with respect to $R$ equal to zero provides us with the functional equation which we must solve for $\alpha(R)$ to give the shape of the distorted disc,

$\frac{\mathrm{d} \omega_{p}(R)}{\mathrm{d} R}[\alpha(R)]=0$.

The functional Eq. (12) can be solved numerically using the method explained in Appendix A. From this, we can obtain $\alpha(R)$, i.e. the amplitude of the warp as a function of the distance from the Galactic centre for a given external torque $\tau_{\text {ext }}$.

\subsubsection{Few remarks on the transient regime towards the equilibrium configuration}

As explained above, we are not concerned here about the transient regime. However, it is interesting to comment on the qualitative aspects of this regime, since they indicate that some of the mechanisms considered here may well be of interest. For a mechanism to be acceptable it should lead to a stationary result, in reasonable agreement with the observations, which are assumed to correspond to stationary systems. However the mechanism is not plausible 


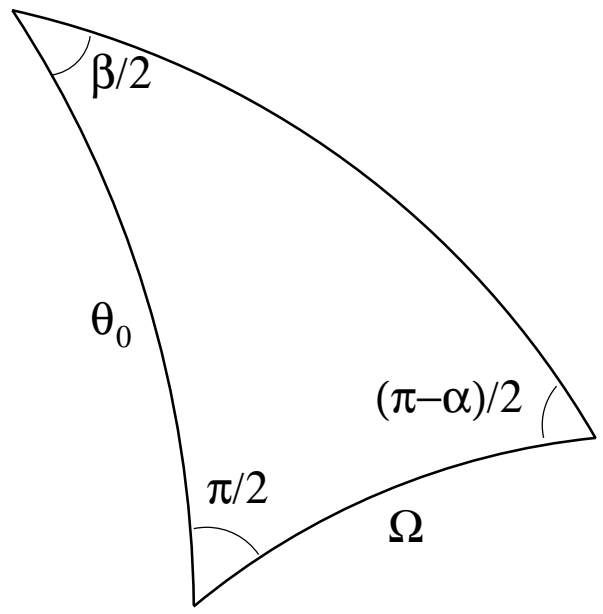

Fig. 1. Spherical triangle which relates different parameters in differential precession of two rings.

unless it offers a credible scenario for the formation of a warp once the external influence initiates its operation. We will now see how qualitative considerations relative to the warp formation process do indicate different degrees of plausibility for different mechanisms, even where their predictions for the stationary state are similar.

To carry through this qualitative analysis, it is useful to consider Eqs. (10) for a case in which all the mass is concentrated in two rings (orbits), one of these being much more massive than the other. $\boldsymbol{\tau}_{\text {ext }}$ is always perpendicular to $\boldsymbol{u}, \boldsymbol{k}$ (although this does not hold for $\boldsymbol{\tau}_{\text {int }}$ ), and since initially $\boldsymbol{\tau}_{\text {int }}=\mathbf{0}$ the initial motion is a pure precession of both rings around $\boldsymbol{u}$. However this precession occurs, in general, at a different rate for each ring. So the plane of one of the rings will be elevated "above" the plane of the other, at a dihedric angle $\alpha$. After they have precessed differentially through an angle $\beta$, and the line of nodes forms an angle $\Omega$ with the projection of the vector $\boldsymbol{u}$ onto the plane of the more massive ring, the relation between these three quantities and $\theta_{0}\left(\right.$ where $\left.\sin \theta_{0}=\boldsymbol{k} . \boldsymbol{u}\right)$ is given implicitly by the spherical triangle in Fig. 1. Clearly as $\beta$ goes to zero, $\Omega$ also goes to zero. This means that the initial node direction is at $\pi / 2$ with respect to the final stationary position. Now as $\alpha$ increases $\tau$ increases, producing an additional precession of the plane of the lighter ring around the vector perpendicular to the plane of the heavier.

If the light ring precesses initially around $\boldsymbol{u}$ faster than the heavy ring (this holds in the general case for rings with an initial precession velocity faster than the mean), the additional precession induced in the heavy ring is in the sense opposed to $\dot{\Omega}$. So this additional precession must sweep out an angle somewhat greater than $\pi / 2$ before it reaches the stationary state. If the light ring precesses initially more slowly the corresponding angle would be somewhat less than $\pi / 2$. However the implication is that the time required to reach the stationary condition is of order a quarter of the initial precession period.
Translating this to the real case, it implies that the time for the formation of the warp must be of the order of one quarter of the final global precession period. This time could be quite long, and a formation procedure which leads to a shorter time scale would be more plausible. This is the case, for example, where $\boldsymbol{\tau}_{\text {ext }}$ is absorbed by the gas. In this case to simulate the evolution we must consider Eq. (10) for two sets of rings, gas rings, which are affected by both $\boldsymbol{\tau}_{\text {ext }}$ and $\boldsymbol{\tau}_{\text {int }}$, and stellar rings, which are affected only by $\boldsymbol{\tau}_{\text {int }}$. Qualitatively the gas rings will move initially as indicated above, giving rise to a $\boldsymbol{\tau}_{\text {int }}$ which is perpendicular to $\tau_{\text {ext }}$. The stellar rings which initially remain in the same plane start to move under the influence of $\boldsymbol{\tau}_{\text {int }}$, with $\dot{\boldsymbol{k}}(R, t)$ parallel to the projection of $\boldsymbol{u}$ onto the plane of the ring so that the resulting nodes already are in their final position. For very large radii $R$, the rings would precess in a direction perpendicular to that for the inner rings because the torque of the inner stellar rings, which are already warped, dominate that due to the gas. Thus, the line of the nodes of these orbits will form some angle (going asymptotically to $\pi / 2$ ) with that of the inner orbits. The nodes of the outer orbits trace a leading spiral. This seems to agree qualitatively with some observations (Briggs 1990).

The gas rings then move rapidly under the much stronger $\boldsymbol{\tau}_{\text {int }}$ generated by the stellar rings, and align generally with them, although the final warp will be slightly different for the gas rings and the stellar rings, so that the $\tau_{\text {ext }}$ experienced by the gas is transferred gravitationally almost entirely (but not quite) to the stellar ring at the same radius, although probably the relative displacement between the two is smaller than the thickness of the disc, and will not be considered in the present paper.

As we pointed out earlier, Eq. (10) is first order, so if when the orbits arrive at their stationary position the torque takes their stationary values (which happens when all orbits reach their stationary positions simultaneously) the line of the nodes of every orbit will stop precessing in the mean plane defined by all other orbits, so stationarity is achieved. However this does not seem likely and a more probable outcome is that the line of the nodes oscillates around the equilibrium position. Perhaps, friction could damp the amplitude of these oscillation. There is no problem in this oscillation being eliminated without dissipation (since they contain no energy) but it seems unlikely because of the degree of conspiracy between the orbits required. Thus our stationary solution will correspond to the statistical mean, but at any time there will be present minor wiggles (in $\alpha(R)$ ) superimposed on it.

The comment on warp formation presented here is a qualitative anticipation of future detailed work and does not affect any of the conclusions of the present work.

\subsection{Example of application: gravitational torque}

Once we have a general formalism in which a warp is generated when a torque is produced in a galactic disc, we 


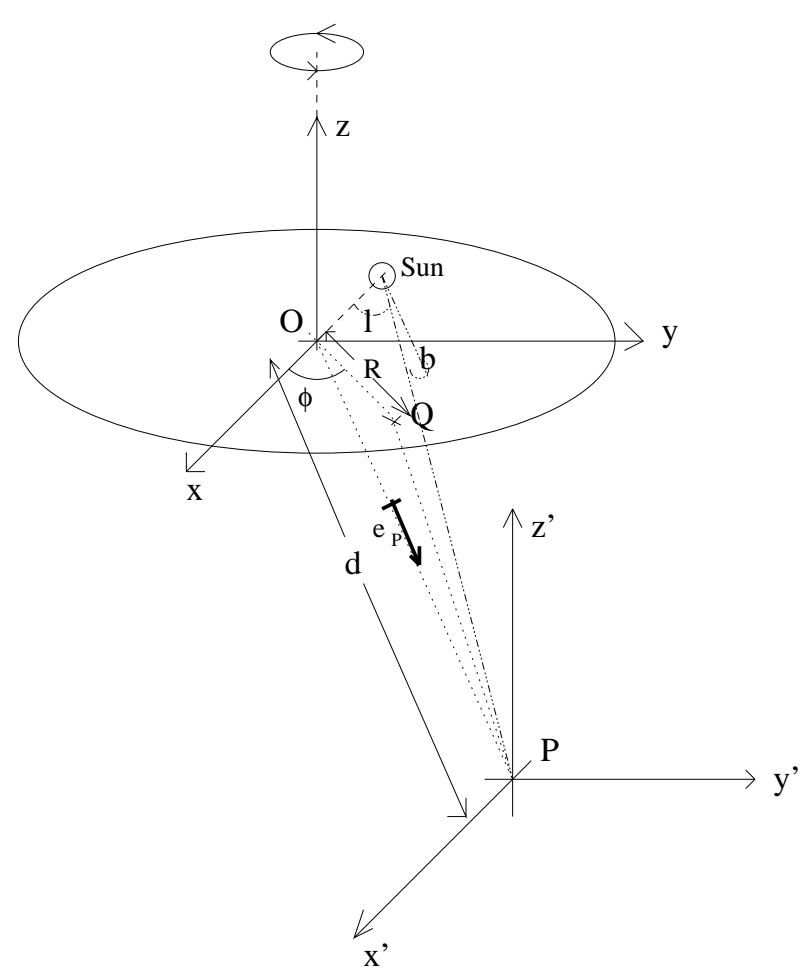

Fig. 2. Graphical representation of the Galactic disc (with coordinates $x, y, z$ ) and a point mass at $P$ (with coordinates $\left.x^{\prime}, y^{\prime}, z^{\prime}\right)$.

can analyze possible mechanisms to produce that torque. A conventional example is the torque generated by gravitational tidal effects.

In Fig. 2, we show the Galactic disc centred at $O$ and a point mass at $P$ such that $\overline{O P}=d \boldsymbol{e}_{\boldsymbol{P}}$. In the case of the Milky Way disc, the Sun would be situated in the disc with $R=R_{0}, \phi=180^{\circ}$. The unit vector can be expressed as

$\boldsymbol{e}_{\boldsymbol{P}}=\cos \phi_{P} \cos \theta_{P} \boldsymbol{i}+\sin \phi_{P} \cos \theta_{P} \boldsymbol{j}+\sin \theta_{P} \boldsymbol{k}$.

It is well-known that the torque produced by a point mass of mass $m_{P}$ on any axisymmetric body is, considering only the quadrupolar term,

$\tau_{\text {grav }} \approx \frac{3 G m_{P}\left(I_{3}-I_{1}\right)}{2 d^{3}} \cos \theta_{P} \sin \theta_{P}$

$\times\left(\sin \phi_{P} \boldsymbol{i}-\cos \phi_{P} \boldsymbol{j}\right)$,

where $I_{1}$ and $I_{3}$ are the inertia tensor components (in this case for the disc).

The solution of (12) with $\tau_{\text {ext }}=\tau_{\text {grav }}$ by means of the numerical method explained in Appendix A gives $\alpha(R)$ for the galactic warp. It will depend on the adopted Galactic model. For the Milky Way, a suitable model can be adopted as follows:

The surface density of the disc, (9), is taken as

$\sigma(R)=\left\{\begin{array}{ll}\sigma\left(R_{0}\right) \mathrm{e}^{-\frac{R-R_{0}}{h_{R}}}, & R \leq 3 R_{0} \\ 0, & R>3 R_{0}\end{array}\right\} ;$

where the local surface density is $\sigma\left(R_{0}\right)=48 M_{\odot} \mathrm{pc}^{-2}$ (Kuijken \& Gilmore 1989), the distance to the Galactic

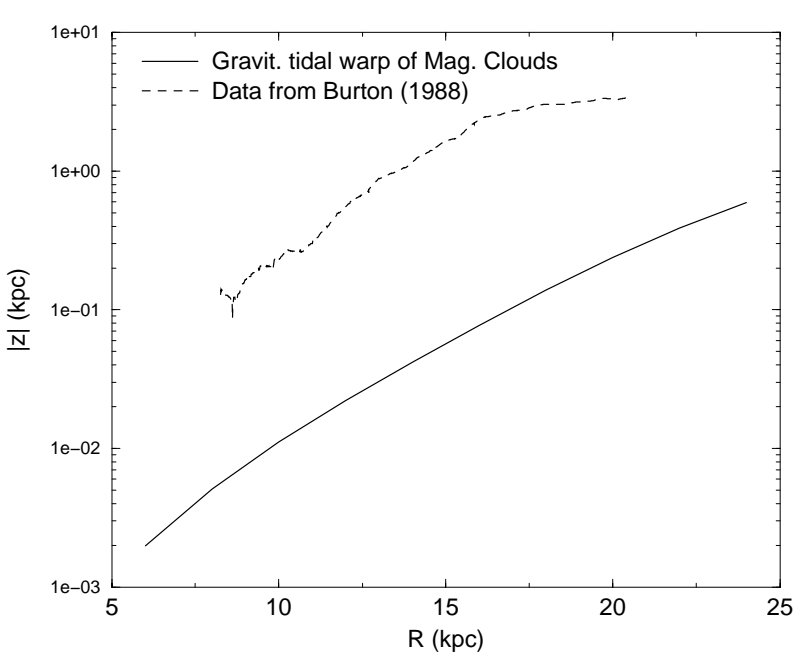

Fig. 3. Milky Way warp maximum height as a function of the radius due to gravitational tidal effects of the Magellanic Clouds (solid line). Dashed line stands for observational data of the northern warp (Burton 1988) rescaled to $R_{0}=7.9 \mathrm{kpc}$.

centre is $R_{0}=7.9 \mathrm{kpc}$ (López-Corredoira et al. 2000); and the scale length $h_{R}=3.5 \mathrm{kpc}$ (Bahcall \& Soneira 1980). We truncate the exponential disc at $3 R_{0}$. The Galactic disc undoubtedly extends to larger radii but the effects of those outer rings can be considered negligible in the present dynamical context.

The rotation velocity is taken as

$v_{\text {rot }}(R)=R \omega_{\text {rot }}(R)$

$=\left\{\begin{array}{ll}200 \mathrm{~km} \mathrm{~s}^{-1}, & R \leq 15 \mathrm{kpc} \\ 200 \sqrt{15 \mathrm{kpc} / R} \mathrm{~km} \mathrm{~s}^{-1}, & R>15 \mathrm{kpc}\end{array}\right\}$.

(Honma \& Sofue 1996). Outside the stellar truncation radius $(\sim 15 \mathrm{kpc})$, a Keplerian law $\left(v_{\text {rot }} \propto R^{-1 / 2}\right)$ is followed, which implies that we have neglected dark matter contributions from larger radii (according to Honma \& Sofue 1996, there is no essentially dark matter beyond $15 \mathrm{kpc}$ ).

For the Large Magellanic Cloud, we adopt $\theta_{P}=-33^{\circ}$, $m_{P}=10^{10} M_{\odot}$ and $d=55 \mathrm{kpc}$, the same values that Hunter \& Toomre (1969) adopted.

The precession we obtain is $\omega_{p}=2.7 \times 10^{-19} \mathrm{rad} / \mathrm{s}$ and the amplitude of the warp is shown in Fig. 3 where $\alpha(R) \approx|z| / R$. The functional shape of $\alpha(R)$ resembles that of observational data, but the amplitude is very different: a factor of 20 or 30 separates the two curves, in agreement with Hunter \& Toomre (1969). This means that we would require the mass of the Magellanic Clouds to be around 2 or $3 \times 10^{11} M_{\odot}$ to justify the Milky Way warp as a product of the gravitational interaction with the Clouds. The calculation of the amplitude with the Saggitarius dwarf galaxy $\left(m_{P}=10^{9} M_{\odot}, d=16 \mathrm{kpc}\right.$; Ibata \& Razoumov 1998) gives an amplitude 4 or 5 times larger, which is still not enough to produce the warp, nor is the predicted direction of the warp in agreement with the observations. 


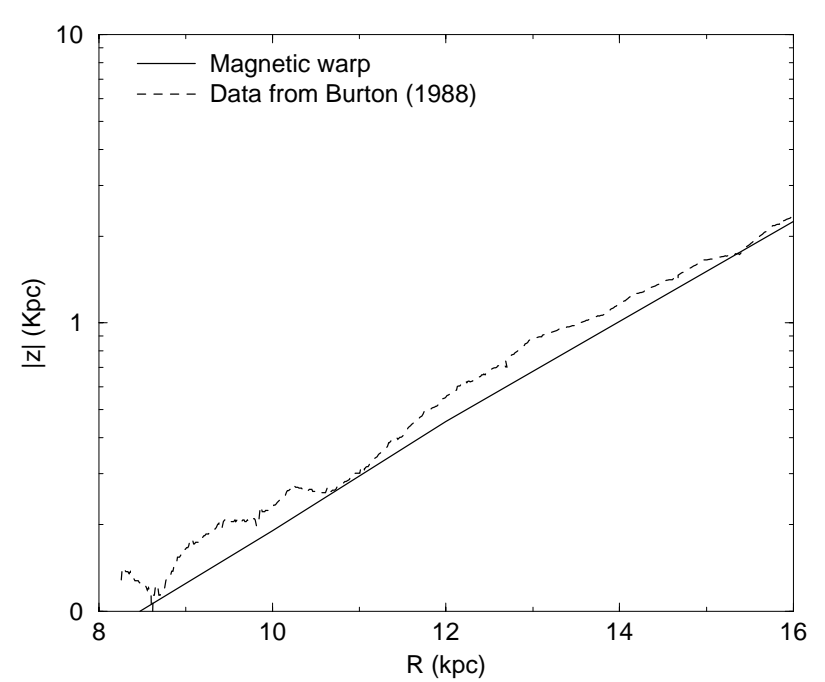

Fig. 4. Milky Way warp maximum height as a function of the radius due to an extragalactic magnetic field of intensity $1.4 \mu \mathrm{G}$ (solid line). Dashed line stands for observational data of the northern warp (Burton 1988) rescaled to $R_{0}=7.9 \mathrm{kpc}$.

\subsection{Example of application: Magnetic torque}

Another example can be calculated: the case of magnetic forces (Battaner et al. 1990; Battaner \& Jiménez-Vicente 1998), in which the force per unit volume which produces the torque for a $\theta_{P}=45^{\circ}$ inclination field is:

$\boldsymbol{F}=\frac{B^{2} \sin \left(2\left(\theta_{P}-\alpha(R)\right)\right.}{16 \pi L} \sin \left(\phi-\phi_{P}\right) \boldsymbol{k}$,

where $L=1 \mathrm{kpc}$ is the adopted value for the characteristic length in which galactic regions dominated by the galactic magnetic field become dominated by the extragalactic magnetic field. Hence, the torque over a ring between $R$ and $R+\mathrm{d} R$ is:

$\boldsymbol{\tau} \mathrm{d} R=2 h_{z} R \mathrm{~d} R \int_{0}^{2 \pi} \mathrm{d} \phi \boldsymbol{r} \times \boldsymbol{F}$

$=\frac{B^{2} R^{2} h_{z} \sin \left(2\left(\theta_{P}-\alpha(R)\right)\right) \mathrm{d} R}{8 L}\left(\sin \phi_{P} \boldsymbol{i}-\cos \phi_{P} \boldsymbol{j}\right)$,

where $h_{z}=0.1 \mathrm{kpc}$ is the scale height of the disc.

With the same Galactic model as the previous subsection, we obtain that the amplitude of the magnetic field is

$B \sim 1.4 \mu \mathrm{G}$,

in order to obtain a coincidence with observational Burton (1988) data. The precession angular velocity is $\omega_{p}=5.1 \times$ $10^{-18} \mathrm{rad} / \mathrm{s}$.

There is a good agreement for the curves in Fig. 4, which was noted by Battaner \& Jiménez-Vicente (1998). The intensity of the field required to produce the warp is perhaps somewhat high, but we will not discuss in this paper the possibility of the existence of such a field. Kronberg (1994), for instance, argues that the value of the intergalactic magnetic field can be as high as this. We remark only that the possibility of warps generated by extragalactic magnetic fields should not be taken lightly, although the high value on the required field is perhaps questionable. This value is in agreement with that of Battaner \& Jiménez-Vicente (1998) although their calculation method is much simpler. Binney (1991) obtained a higher required value for $B$, with a difference of an order of magnitude, in part due to his adoption of an expression for the magnetic force different from expression (17) equivalent to a much lower value of $L$. He also adopted an insufficiently precise approximation of an axisymmetric potential not distorted by the warp.

\section{Generation of warps by an intergalactic flow}

\subsection{Accretion of matter onto the galactic disc and warps}

The idea we are suggesting here is not totally new. It is based on ideas about infall of intergalactic matter previously explored by other authors (Kahn \& Woltjer 1959; Binney \& May 1986; Ostriker \& Binney 1989; Jiang \& Binney 1999). Kahn \& Woltjer (1959) first suggested that an intergalactic matter flow could bring about the warp; however, their rough calculations in fact derive a pressure gradient transmitted to the disc by means of a hypothetical halo compressed by a subsonic massive wind and do not specify the mechanism of generation of the warp. The representation they use is very simple, and quite different from that presented here. The dominant response to the intergalactic medium ram pressure of a disc moving through it would be axisymmetric, taking the form of a rim rather than a warp (Binney 1992), although Kahn \& Woltjer's estimates of the amplitude in fact agree with our values and their work was a first indication of a possible mechanism to explain warps. Ostriker \& Binney's (1989) suggestion of how cosmic infall can bring about warped galactic discs is rather qualitative and they did not proceed to a quantitative analysis. Subsequent papers (Binney et al. 1998; Jiang \& Binney 1999) use a model based on the infall onto the Galactic halo and do not consider the direct infall onto the disc. The latter is the novelty here: we take the idea of cosmic infall, and explore the torque it creates when it collides transmitting its angular momentum, but we consider directly the interaction with the disc. We do not invoke the idea of a massive halo modulating the dynamical effects on the disc.

The idea of cosmic infall has been considered in the context of CDM theory. Ryden \& Gunn (1987) and Ryden (1988) have shown that half of the total angular momentum of any galaxy was contributed by material that fell in over the last third of a Hubble time. Galaxy formation theories require this infall. Furthermore, many observations imply that there must be an infall of material to galaxies (Binney 2000): Local Group members approach each other, the high velocity clouds around the Galaxy have on the average a net negative velocity (Blitz et al. 1999; Braun \& Burton 1999), and others.

There are good reasons to believe that the infalling baryonic matter is accreted directly by the disc rather 
than the halo (non-baryonic matter can escape completely or be captured by the halo). The principal supporting arguments here are those based on the observed chemical evolution (Ostriker \& Binney 1989; López-Corredoira et al. 1999). Significant accretion of metal poor gas is necessary to justify the observations concerning star formation and metallicity distribution in the galactic disc, often termed the G-dwarf problem (Tinsley 1980) and the details of time-dependent evolution of individual metals (Casuso \& Beckman 1997, 2000). Recent results implying that this accretion has been constant, or even increased, during the disc lifetime, are found in Rocha-Pinto et al. (2000). Moreover, it is clear that a halo should trap accreted matter with low efficiency, since its mean baryonic density is very low.

In a more general context, it should not be thought that the general secular infall of matter is the only factor. Any cloud in the intergalactic medium whose orbit intersects the galaxy and is accreted by the disc provides a torque due to the interchange of its angular momentum. For instance, the exchange of matter between two galaxies can supply accretable intergalactic matter: an intergalactic flow is produced between the two galaxies. The HVCs (High Velocity Clouds) have been suggested (Blitz et al. 1999; López-Corredoira et al. 1999; Wakker et al. 1999a; Binney 2000) to be observable evidence of the material which is continuously falling onto the Galactic disc.

\subsection{Description of the flow}

An intergalactic flow can be described as a beam of particles which comes from infinite distance towards the galactic disc with velocity $\boldsymbol{v}_{\mathbf{0}}$. Each particle of the beam follows a trajectory which is not a straight line, due to the gravitational attraction of the galaxy, until it reaches the galactic plane $(z=0)$. As it intersects the plane, it collides with the gas of the galaxy and remains trapped in the disc. A torque results from the angular momentum contribution of this particle to the disc. The net torque over a ring of the disc of radius between $R$ and $R+\mathrm{d} R$ will be the sum of the torques produced by all the particles of the beam which collide with the galactic disc at distance between $R$ and $R+\mathrm{d} R$ from its centre.

The total angular momentum with respect to the centre of the galaxy which is transported by a cylindrical beam of these characteristics with axis crossing the centre of the galaxy is zero. Is the total angular momentum deposited in each ring also zero? The answer to this question is "no", and this is the key to warp generation by an intergalactic flow. The net momentum transferred to each ring is non-zero because, for a general case where the net flow is not perpendicular to the plane of the galaxy nor isotropic, the particles which fall onto a given ring do not come from a single cylindrical shell of the flow. The particles are redistributed, and so is their angular momentum, due to the gravitational interaction with the galaxy. Therefore, the impact parameter of each infalling particle

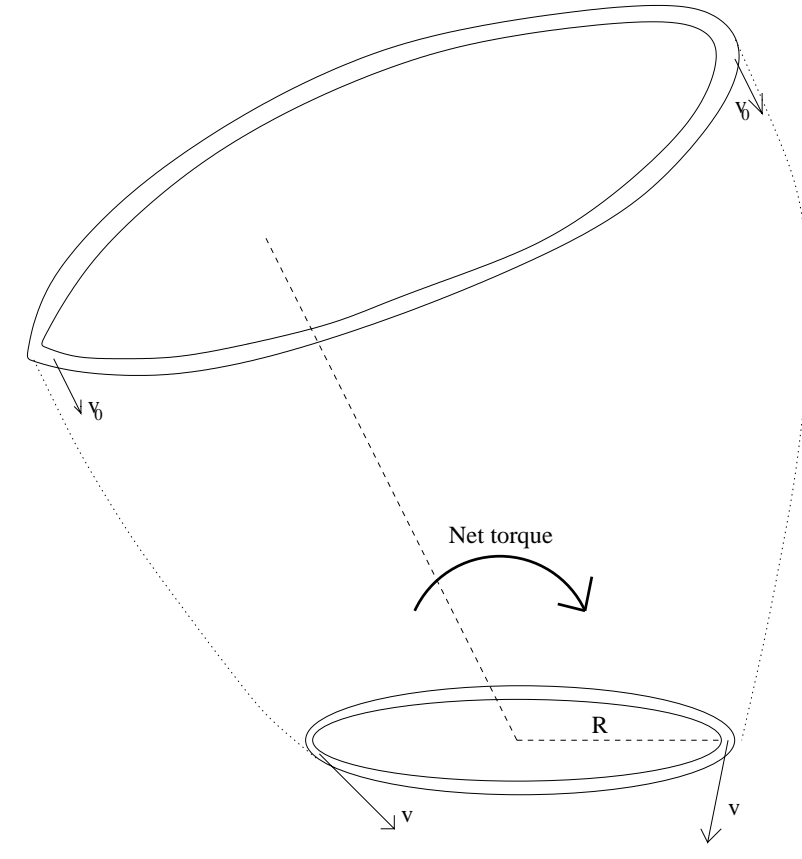

Fig. 5. Graphical representation of the infall of an intergalactic flow (non-circular ring, above) which produces a net torque on a circular ring in the galactic disc (below).

is not the same for all the particles which cut a given ring (if it were the same the net contribution of the angular momentum would indeed be zero); this will be analytically expressed in the equations given below in the present section. In particular, the variation of the impact parameter for a ring as a function of azimuthal angle is expressed in Eq. (29).

The calculations in subsections below are, perhaps, a little complicated to follow. However, the qualitative description of the physical system whose variables we will calculate is not difficult to understand. Figure 5 gives a pictorial description which should be helpful. There is a net torque because the set of particles which fall into the ring between radii $R$ and $R+\mathrm{d} R$ comes from a non-circular ring (which is not in fact elliptical). The transformation of this non-circular ring to the circular ring in the disc plane is effected by gravity. The velocity of the impacting particles varies with azimuth in the galactic plane ring. We wish to calculate the total angular momentum which is transported by matter in the non-circular ring which falls into the circular ring. Conservation of angular momentum implies that the angular momentum transmitted to the circular ring must be the same as that in the noncircular ring. Nevertheless, calculation of this angular momentum is not easy because the geometrical shape of the non-circular ring is not easy to describe analytically. This gives rise to the rather tedious calculations in subsections below.

Once a torque is produced over each ring a warp is generated to compensate the differential precession of the rings, as we have seen in the previous section. We have explained the mechanism of generation of warps when any 


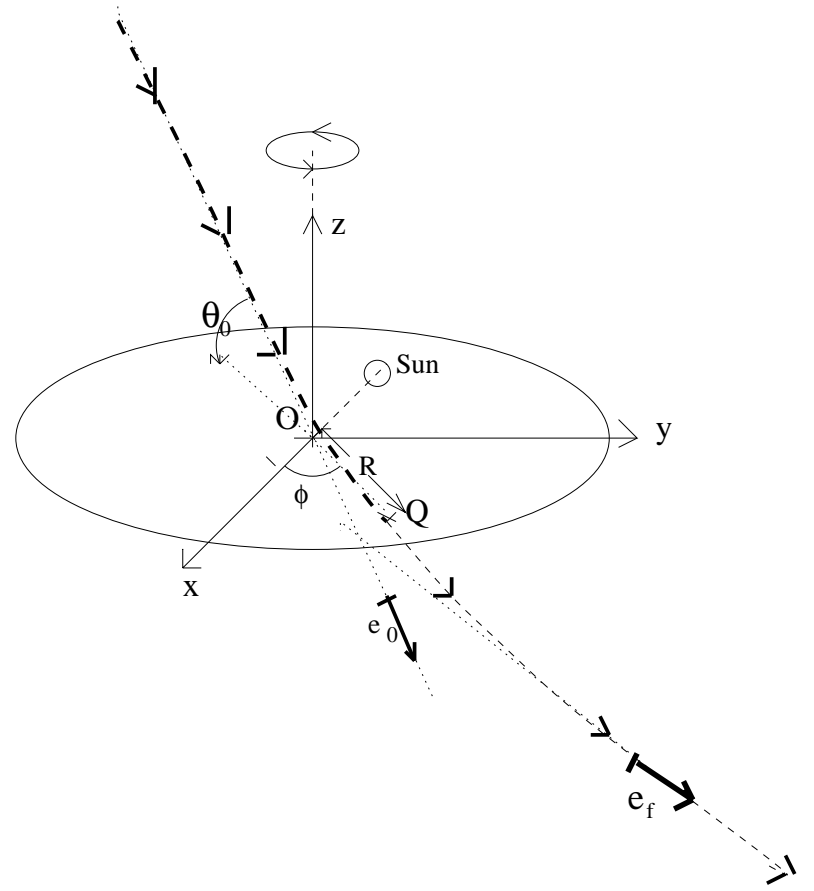

Fig. 6. Graphical representation of the Galactic disc in the coordinates system $x y z$, and the hyperbolical trajectory of an infall particle.

kind of torque due to external forces is applied over the disc, in Sect. 2. Now, in this section, we carry out the calculation of the torque due to the intergalactic flow.

\subsection{Torque due to collision with a particle}

In Fig. 6, we give a graphical representation of the galactic disc centred at $O$, and the trajectory of a particle which intersects the disc. A particle comes from infinite distance with velocity $v_{0}$ in the normalized direction $\boldsymbol{e}_{\mathbf{0}}$ given by angles $\phi_{0}, \theta_{0}$ in spherical coordinates (in Fig. 6, $\theta_{0}<0$ ), where

$\boldsymbol{v}_{\mathbf{0}}=v_{0}\left(\cos \phi_{0} \cos \theta_{0} \boldsymbol{i}+\sin \phi_{0} \cos \theta_{0} \boldsymbol{j}+\sin \theta_{0} \boldsymbol{k}\right)$,

and it follows a trajectory which crosses the Galactic disc in some point $Q$ whose distance from the centre is $R$ and angle with respect to the $x$-axis (defined as the line "Galactic centre-Sun" with $x$ negative towards the Sun) is $\phi$, i.e.

$\overline{O Q}=\boldsymbol{R}=R \cos \phi \boldsymbol{i}+R_{Q} \sin \phi \boldsymbol{j}$.

A minor order correction for small warp amplitudes, that we also take into account, is the variation of angle of the flow in the warped rings. We must bear in mind that

$\theta_{0}(R)=\theta_{0}(R=0)-\alpha(R)$.

The same trajectory is represented in Fig. 7 in the plane of the orbit $x^{\prime} y^{\prime}$. It is assumed that the trajectory is a hyperbola typical of a two-body gravitating system where the heavier body is the galaxy whose mass is $M_{\text {gal }}$ concentrated at the point $O$. Some minor effects due to the

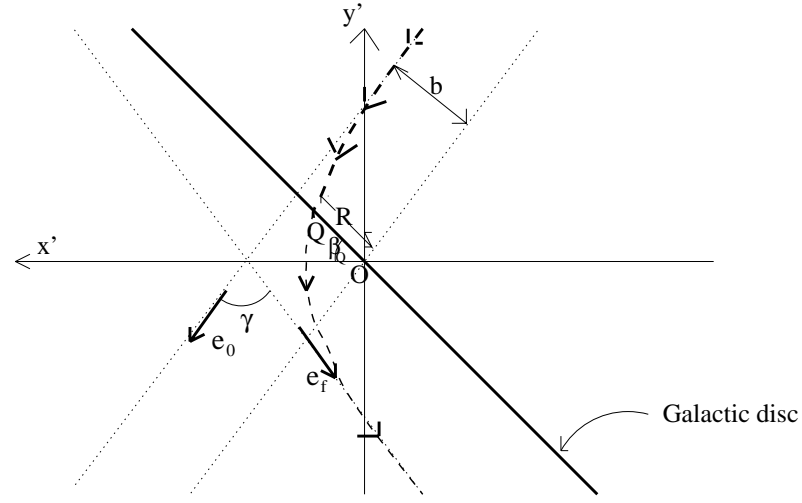

Fig. 7. Graphical representation of the hyperbolical trajectory of an infall particle in the plane of the orbit.

dispersion of the mass throughout the disc are expected but they are negligible if $R$ is larger than several disc scale lengths (i.e. greater than $R \approx 10 \mathrm{kpc}$ ). The orbit is a hyperbola because the energy of the system is positive, since the velocity at infinite distance, $\left|\boldsymbol{v}_{\mathbf{0}}\right|$, is greater than zero. Therefore, the plane of the orbit will be determined by the independent vectors $\boldsymbol{r}$ and $\boldsymbol{v}_{\mathbf{0}}$ and the equation of the orbit in the $x^{\prime} y^{\prime}$ system is $(r$ and $\beta$ are the polar coordinates in this system; see Fig. 7):

$\frac{\epsilon}{A r}=1+\epsilon \cos \beta$,

where $\epsilon$ is the eccentricity of the orbit,

$\epsilon=\sqrt{1+\left(\frac{b v_{0}^{2}}{G M_{\text {gal }}}\right)^{2}}$,

and

$A=\sqrt{\left(\frac{G M_{\mathrm{gal}}}{v_{0}^{2} b^{2}}\right)^{2}+\frac{1}{b^{2}}}=\frac{v_{0}^{2}}{G M_{\mathrm{gal}}} \frac{\epsilon}{\epsilon^{2}-1}$.

The impact parameter is $b$ and the net asymptotic angular deviation $\gamma$ (see Fig. 7) is given by

$\tan \frac{\gamma}{2}=\frac{1}{\sqrt{\epsilon^{2}-1}}=\frac{G M_{\mathrm{gal}}}{b v_{0}^{2}}$

The determination of the point of intersection of the orbit with the disc of the galaxy is a simple trigonometric problem. From a triangle shown in Fig. 7, we can derive:

$\beta_{Q}=\frac{\pi}{2}+\frac{\gamma}{2}-\cos ^{-1}\left(e_{0 Q}\right)$

$e_{0 Q}=\cos \left(\boldsymbol{v}_{\mathbf{0}}, \boldsymbol{r}_{\boldsymbol{Q}}\right)=\frac{\boldsymbol{v}_{\mathbf{0}} \boldsymbol{r}_{\boldsymbol{Q}}}{v_{0} r_{Q}}=\cos \left(\theta_{0}\right) \cos \left(\phi_{0}-\phi\right)$.

From these expressions, together with (23), (24), (25) and (26), the radial galactocentric distance $R$ of the point of orbit intersection with the Galactic plane is:

$R=r_{Q}=\frac{b^{2} v_{0}^{2}}{b v_{0}^{2} \sqrt{1-e_{0 Q}^{2}}+G M_{\mathrm{gal}}\left(1-e_{0 Q}\right)}$. 
The angular momentum of the particle with mass $\mathrm{d} m$ is constant along its trajectory,

$\boldsymbol{J}=\mathrm{d} m v_{0} b \frac{\boldsymbol{r}_{\boldsymbol{Q}} \times \boldsymbol{v}_{\mathbf{0}}}{r_{Q} v_{0}\left|\sin \left(\boldsymbol{v}_{\mathbf{0}}, \boldsymbol{r}_{\boldsymbol{Q}}\right)\right|}$,

and the torque produced by a particle which transmits its angular momentum to the disc is:

$\boldsymbol{\tau}=\frac{\mathrm{d} \boldsymbol{J}}{\mathrm{d} t}=\frac{\mathrm{d} m}{\mathrm{~d} t} v_{0} b \frac{\boldsymbol{r}_{\boldsymbol{Q}} \times \boldsymbol{v}_{\mathbf{0}}}{r_{Q} v_{0} \sqrt{1-e_{0 Q}^{2}}}=\frac{\mathrm{d} m}{\mathrm{~d} t} v_{0} b\left(1-e_{0 Q}^{2}\right)^{-1 / 2}$

$\times\left[\sin \phi \sin \theta_{0} \boldsymbol{i}-\cos \phi \sin \theta_{0} \boldsymbol{j}+\cos \theta_{0} \sin \left(\phi_{0}-\phi\right) \boldsymbol{k}\right]$.

The new material is stopped by the friction with the disc and its angular momentum added to the ring. Note that although the ring is not really a rigid body it behaves like one. A single particle in orbit around the Galactic centre can resemble the dynamics of the rigid body; it carries the increase of angular momentum and the orbit is distorted according to the applied torque.

The flow is stopped by the friction with the gas, so the angular momentum is, at first, transmitted to the gas. This does not mean that gas disc warps while stellar disc does not. Indeed, it is expected that stars in the ring feel the gravitational torque due to the gas rings and are dragged by them. There may be some difference between the stellar warp and the gas warp due to this lag in the dynamics, but the difference is likely to be small. If the stellar disc were demonstrated to be less warped than the gas warp, it would be evidence in favour of either this theory or the theory of the intergalactic magnetic field as the generator of the warp (Porcel et al. 1997), or indeed of any theory in which the external torque affects directly the gas disc rather than the stellar disc.

\subsection{Total torque due to collision with a particle beam}

Expression (31) gives the torque produced by a particle which falls to the disc with an impact parameter $b$ and intersects the disc at $\boldsymbol{r}_{\boldsymbol{Q}}$. If we want to know the total torque produced by all the particles which come with any $b$ and intersect the disc at a distance between $R$ and $R+\mathrm{d} R$ with any azimuth $\phi$, we have to integrate over all the particles of the beam which fall in this ring. The whole beam is then represented by the varying in the plane perpendicular to $\boldsymbol{v}_{\mathbf{0}}$ the initial (at infinite distance) position of the falling particle, whose polar coordinates are $b$ and $\phi_{b}$ (see Fig. 8). Thus, the total torque exerted over the ring with radius between $R$ and $R+\mathrm{d} R$ is

$$
\begin{aligned}
& \boldsymbol{\tau}(R) \mathrm{d} R=\int_{0}^{2 \pi} \mathrm{d} \phi_{b} \int_{0 ; R<R_{Q}<R+\mathrm{d} R}^{\infty} \mathrm{d} b b \\
& \times\left[\frac { \mathrm { d } m } { \mathrm { d } t } v _ { 0 } b ( 1 - e _ { 0 Q } ( \phi ) ^ { 2 } ) ^ { - 1 / 2 } \left[\sin \phi \sin \theta_{0} \boldsymbol{i}\right.\right. \\
& \left.\left.-\cos \phi \sin \theta_{0} \boldsymbol{j}+\cos \theta_{0} \sin \left(\phi_{0}-\phi\right) \boldsymbol{k}\right]\right], \\
& \mathrm{d} m=\rho_{\mathrm{b}} v_{0} \mathrm{~d} t,
\end{aligned}
$$

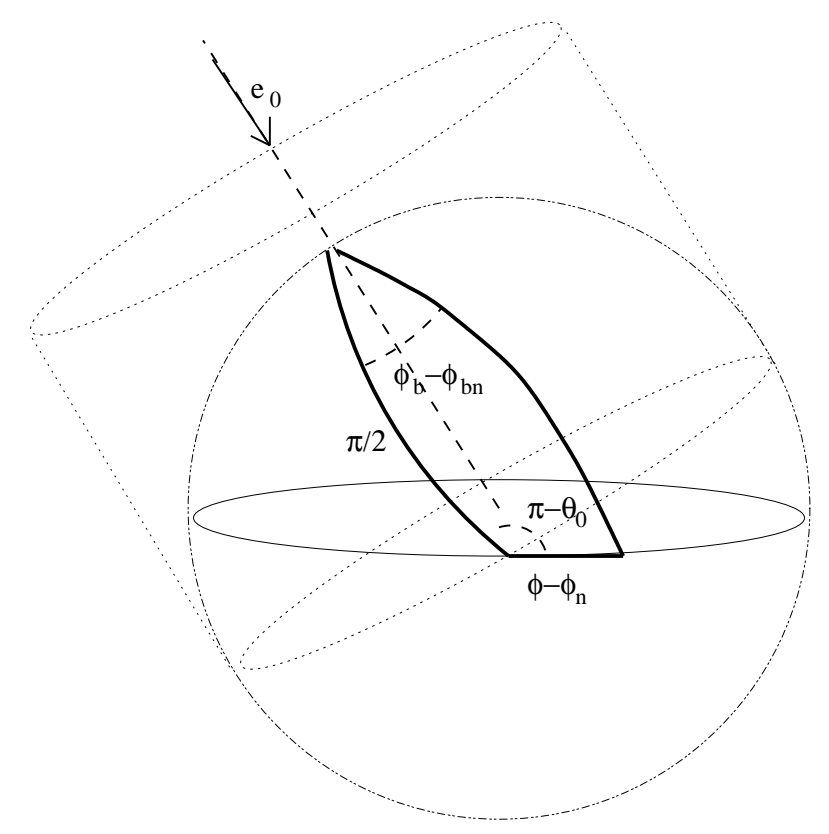

Fig. 8. How the polar angles $\phi_{b}$ in the original flow and $\phi$ in the plane of the galaxy are related in terms of a spherical triangle.

where $\rho_{\mathrm{b}}$ is the density of baryonic matter in the particle beam, assumed to be independent of $b$ and $\phi_{b}$. Any nonbaryonic matter in the inflow would not be trapped in the disc so it should not be taken into account within the total mass of the flow for the purpose of computing the torque. In the notation, $\tau$ stands for the torque per unit galactocentric radial length. Note that $\phi_{b}$ is the polar angle in the plane perpendicular to $\boldsymbol{v}_{\mathbf{0}}$ and is different from the polar angle $\phi$ in the Galactic disc. The relationship between the two is:

$\cot \left(\phi_{b}-\phi_{b n}\right)=\frac{\cot \left(\phi-\phi_{n}\right)}{\left|\sin \theta_{0}\right|}$

according to the general formula relating the angles in the spherical triangle of Fig. 8. $\phi_{n}$ and $\phi_{b n}$ are the polar angles of the galactic disc and the plane perpendicular to $\boldsymbol{v}_{\mathbf{0}}$ respectively corresponding to the node where the two planes intersect. We can choose for convenience the origin of the angles $\phi_{b}$ such that:

$\phi_{b n}=0$,

and the angle of the line of nodes in the galactic disc is:

$\phi_{n}=\phi_{0} \pm \pi / 2$.

We change the variables of integration in the expression (32) to $R$ and $\phi$ (the Jacobian of the transformation is $\frac{\partial \phi_{b}}{\partial \phi} \frac{\partial b(R, \phi)}{\partial R}$; we neglect $\frac{\partial \phi_{b}}{\partial R}$ due to the dependence $\left.\theta_{0}(R)\right)$ and obtain

$\boldsymbol{\tau}(R)=\frac{\rho_{\mathrm{b}} v_{0}^{2}}{\left|\sin \theta_{0}\right|} \int_{0}^{2 \pi} \frac{\mathrm{d} \phi\left(1-e_{0 Q}(\phi)^{2}\right)^{-1 / 2}}{1+\sin ^{2}\left(\phi_{0}-\phi\right)\left(\sin ^{-2} \theta_{0}-1\right)}$

$\times\left[\sin \phi \sin \theta_{0} \boldsymbol{i}-\cos \phi \sin \theta_{0} \boldsymbol{j}+\cos \theta_{0} \sin \left(\phi_{0}-\phi\right) \boldsymbol{k}\right]$

$\times \frac{\partial b(R, \phi)}{\partial R} b(R, \phi)^{2} ;$ 
where $b(R, \phi)$ is derived from (29):

$b(R, \phi)=\frac{1}{2} R \sqrt{1-e_{0 Q}^{2}(\phi)}$

$+\sqrt{\frac{1}{4} R^{2}\left(1-e_{0 Q}^{2}(\phi)\right)+R G M_{\mathrm{gal}} v_{0}^{-2}\left(1-e_{0 Q}(\phi)\right)}$.

If we make a new change of variables in the integral, substituting $x=e_{0 Q}(\phi)$ for $\phi$ according to (28), and simplify the expression (some terms cancel because of the antisymmetry in the interval $\left(\phi-\phi_{0}\right) \in(0, \pi)$ and $\left.\left(\phi-\phi_{0}\right) \in(\pi, 2 \pi)\right)$, we obtain

$\boldsymbol{\tau}(R)=\frac{2 \rho_{\mathrm{b}} v_{0}^{2} \sin \theta_{0}}{\left|\sin \theta_{0}\right| \cos ^{2} \theta_{0}}\left(\sin \phi_{0} \boldsymbol{i}-\cos \phi_{0} \boldsymbol{j}\right)$

$\times \int_{-\cos \theta_{0}}^{\cos \theta_{0}} \mathrm{~d} x x \frac{\partial b(R, x)}{\partial R} b(R, x)^{2}$

$\times \frac{1}{\sqrt{1-x^{2}} \sqrt{1-x^{2} / \cos ^{2} \theta_{0}}\left[1+\left(1-x^{2} / \cos ^{2} \theta_{0}\right)\left(\sin ^{-2} \theta_{0}-1\right)\right]}$.

The integral is positive. The direction of the torque is $\pm\left(\sin \phi_{0} \boldsymbol{i}-\cos \phi_{0} \boldsymbol{j}\right)$; the sign is "+" when $\theta_{0}<0$ and "-" when $\theta_{0}>0$. That is, the torque is in the disc and perpendicular to $\boldsymbol{v}_{\mathbf{0}}$.

The direction of the infall of a particle flow is the same as that produced by the gravitational torque when $\boldsymbol{e}_{\mathbf{0}}=\boldsymbol{e}_{\boldsymbol{P}}$, although the amplitude is rather different. Note, for instance, that the amplitude in (39) does not depend on the disc density. Therefore, the effect produced by the infall of the particle beam is similar to that due to gravitational effects.

The torques between rings do depend on disc densities (expression (5) is proportional to $\sigma(R) \sigma(S)$ ). This explains why the disc will warp significantly only where its surface density is low (Ostriker \& Binney 1989) which, in practice, means at its outer edge. For the inner disc, $\sigma$ is high enough to provide strong torques between two rings with a small angle $\alpha_{R, S}$ of separation which can compensate the difference of precession with respect to the average. However, external rings must separate further to compensate these differences.

Note that there is no $z$-component of the torque. Taking into account that the total mass of the ring is increased, this means that the angular velocity of rotation should decrease and, therefore the radius of the orbit will be reduced (the increasing mass of the inner galaxy will also tend to reduce the radius) and the galaxy will concentrate further material in the inner regions. We will not study these aspects further in the present paper.

The limit of low initial velocity $\left(\frac{R v_{0}^{2}\left(1+e_{0 Q}\right)}{4 G M_{\text {gal }}} \ll 1\right)$ gives a proportionality

$\lim _{\left(\frac{R v_{0}^{2}\left(1+e_{0 Q}\right)}{4 G M_{\mathrm{gal}}}\right) \longrightarrow 0} \boldsymbol{\tau}(R) \propto \rho_{\mathrm{b}} \frac{\left(G M_{\mathrm{gal}}\right)^{3 / 2}}{v_{0}} R^{1 / 2}$

$\times\left(\sin \phi_{0} \boldsymbol{i}-\cos \phi_{0} \boldsymbol{j}\right)$.
This means that a low velocity beam provides a stronger torque. This effect can be seen due to the increased curvature of the hyperbolic trajectories for low velocities. A ring will then accrete flow particles over a wider range of impact parameters, if the velocity of the beam is lower. In the extreme case of $v_{0}=0$ all the particles fall to the centre, so $R=0$ for all cases with a finite impact parameter and there is no divergence $\left(R \propto v_{0}^{2}\right.$ from (29)). Although we have integrated $b$, the impact parameter, from zero to infinity, in a real case $b$ is limited to a finite value. Expression (40) means that at lower flow velocities the disc accretes particles from a greater fraction of the total stream of particles, and this is the reason why extra angular momentum is deposited. Again, as emphasized above, we must note the angular momentum deposited within a given annulus is due to accretion of particles from a nonaxisymmetric volume of space whose integrated angular momentum is thus non-zero.

\subsection{Mass accreted by the galaxy}

The total accretion rate due to this infall is

$$
\begin{aligned}
& \frac{\mathrm{d} M}{\mathrm{~d} t}=\int_{0}^{2 \pi} \mathrm{d} \phi_{b} \int_{0 ; R<R_{\max }}^{\infty} \mathrm{d} b b \frac{\mathrm{d} m}{\mathrm{~d} t}=\rho_{\mathrm{b}} v_{0} \int_{0}^{2 \pi} \mathrm{d} \phi \frac{\mathrm{d} \phi_{b}}{\mathrm{~d} \phi} \\
& \times\left[\int_{0}^{R_{\max }} \mathrm{d} R b(R, \phi) \frac{\partial b(R, \phi)}{\partial R}\right] \\
& =\frac{-\rho_{\mathrm{b}} v_{0}}{\left|\sin \theta_{0}\right| \cos \theta_{0}} \int_{-\cos \theta_{0}}^{\cos \theta_{0}} d x b\left(R_{\max }, x\right)^{2} \\
& \times \frac{1}{\sqrt{1-x^{2} / \cos ^{2} \theta_{0}}\left[1+\left(1-x^{2} / \cos ^{2} \theta_{0}\right)\left(\sin ^{-2} \theta_{0}-1\right)\right]}
\end{aligned}
$$

This rate is positive since the integral is negative $(b(R,-x)>b(R, x))$.

\subsection{Transfer of linear momentum}

It is important at this stage to consider the transfer of linear momentum. While the transfer of angular momentum produces an integral sign shape (component $m=1$ of the galactic warp), as shown above, the linear momentum produces a cup-shaped deformation of the disk (component $m=0$ of the galactic warp). The numbers in Sect. 4 will show that this effect is indeed quite small, and the $m=1$ component is predominant.

The points of equilibrium between the vertical gravitational force and the vertical force due to transfer of linear momentum give the deformation of the disk. The vertical linear momentum due to the accretion of a particle of mass $\mathrm{d} m$ is:

$p_{z}=\mathrm{d} m v \sin \left(\boldsymbol{R}_{\boldsymbol{Q}}, \boldsymbol{v}\right) \sqrt{1-\cos ^{2} \theta_{0} \sin ^{2}\left(\phi_{0}-\phi\right)}$.

The vector $\boldsymbol{v}$ is the velocity at the impact point of the disc $\left(\boldsymbol{R}_{\boldsymbol{Q}}\right)$. The last two factors account for the projection of the velocity onto the vertical axis. It is not easy to visualize 
the origin of these factors; but they can be understood by reference to Figs. 6 and 7. The radial and azimuthal linear momentum would produce some distortion of the orbits within the disc, which is not the subject of the present paper.

From the conservation of the angular momentum, we have:

$|\boldsymbol{J}|=\mathrm{d} m v_{0} b=\mathrm{d} m v R_{Q} \sin \left(\boldsymbol{R}_{\boldsymbol{Q}}, \boldsymbol{v}\right)$.

From expressions (42) and (43), the vertical force of a particle of mass $\mathrm{d} m$ is:

$F_{z}=\frac{\mathrm{d} p_{z}}{\mathrm{~d} t}=\frac{\mathrm{d} m}{\mathrm{~d} t} \frac{v_{0} b}{R_{Q}} \sqrt{1-\cos ^{2} \theta_{0} \sin ^{2}\left(\phi_{0}-\phi\right)}$.

The total vertical force on all the particles of the beam which fall into the ring of radii $R$ and $R+\mathrm{d} R$ is (from now on, $F_{z}$ stands for the vertical force per unit galactocentric radial length):

$$
\begin{aligned}
& F_{z}(R) \mathrm{d} R=\int_{0}^{2 \pi} \mathrm{d} \phi_{b} \int_{0 ; R<R_{Q}<R+\mathrm{d} R}^{\infty} \mathrm{d} b b \frac{\mathrm{d} m}{\mathrm{~d} t} \frac{v_{0} b}{R_{Q}} \\
& \times \sqrt{1-\cos ^{2} \theta_{0} \sin ^{2}\left(\phi_{0}-\phi\right)} \\
& =\frac{2 \rho_{\mathrm{b}} v_{0}^{2} \mathrm{~d} R}{\left|\sin \theta_{0}\right| \cos \theta_{0}} \int_{-\cos \theta_{0}}^{\cos \theta_{0}} \mathrm{~d} x \sqrt{1+x^{2}-c^{2}} \frac{\partial b(R, x)}{\partial R} \frac{b(R, x)^{2}}{R} \\
& \times \frac{1}{\sqrt{1-x^{2} / \cos ^{2} \theta_{0}}\left[1+\left(1-x^{2} / \cos ^{2} \theta_{0}\right)\left(\sin ^{-2} \theta_{0}-1\right)\right]} .
\end{aligned}
$$

This acceleration is compensated by the vertical gravitational acceleration, so:

$$
\frac{F_{z}(R) \mathrm{d} R}{2 \pi \sigma(R) R \mathrm{~d} R} \approx \frac{G M_{\mathrm{gal}}(R)}{R^{2}} \frac{z}{R},
$$

which implies, for a mass derived from the rotation curve, that

$$
z \approx \frac{F_{z}(R) R}{2 \pi v_{\text {rot }}(R)^{2} \sigma(R)}
$$

This monopolar approximation would be exact if the distribution of mass were spheroidal or elliptical. For the disc, whose contribution is dominant in the torque, the contribution of the gravitational force is different:

$$
\begin{aligned}
& \frac{F_{z}(R)}{2 \pi \sigma(R) R}=G z \int_{0}^{R_{\max }} \mathrm{d} s s \sigma(s) \\
& \times \int_{0}^{2 \pi} \frac{\mathrm{d} \phi}{\left[s^{2}+R^{2}-2 s R \cos \phi+z^{2}\right]^{3 / 2}} \\
& \approx G z \int_{0}^{R_{\max }} \mathrm{d} s s \sigma(s) \int_{0}^{2 \pi} \frac{\mathrm{d} \phi}{\left[s^{2}+R^{2}-2 s R \cos \phi\right]^{3 / 2}} .
\end{aligned}
$$

The last approximation is for $z$ small, compared to $(R-s)$; this is a good approach for large $R$. This is also an approximation in another sense: it does not take into account the distortion of the disc produced by both $m=0$ and $m=1$ components of the warp. In any case, most of the mass is in the inner rings which are not distorted and this effect is small. Hence, $z$ is inversely proportional to the attraction of the disc even for a non-monopolar approximation.

The disc contribution is something larger than its monopolar contribution, so $z$ is lower than (47). This means that the expression (47) gives, approximately, a maximum limit for the distortion of the disc, i.e.

$z \leq \frac{F_{z}(R) R}{2 \pi v_{\text {rot }}(R)^{2} \sigma(R)}$.

This approximation is good enough when the effects of the transfer of linear momentum are nearly independent of those due to the transmission of angular momentum. A more accurate model would refine these approximations and calculate both the $m=0$ and $m=1$ distortions simultaneously. We will see later that the effect of the transfer of linear momentum is small compared with the transfer of angular momentum and, therefore, we do not need to make these more refined calculations for this effect, but only for the integral sign warp, which is dominant.

Is it counterintuitive to have $m=1$ deformation larger than $m=0$ deformation? We can argue that it is not. Imagine a galaxy in which practically all the mass is in the centre, so that the disc has a negligible mass. How big is the countertorque of the galaxy to compensate the external torque? The answer is: zero (or nearly zero), because only the dipole and higher order components of the gravitational attraction produce a countertorque. The monopolar component does not create countertorque. In this case, what will be the equilibrium condition in which the torque is compensated by the countertorques of the disc? It is an " $m=1$ deformation of the disc" (i.e. a warp) whose amplitude tends to infinity (as the mass of the disc tends to zero). In fact the limiting case will not be at infinity, since we are talking about an angular amplitude. The ring will take the angle of incidence of the wind as its maximum limit. So what happens to the " $m=0$ deformation"? This deformation is nothing like as strongly dependent on the distribution of the mass and there is a net finite counterforce of the galaxy to compensate the external force; the amplitude of the $m=0$ cup-shaped distortion is thus finite. Therefore, we have a very high amplitude for $m=1$ and the amplitude of $m=0$ is much smaller. If the density of the wind tends to zero, the amplitude of $m=0$ distortion tends to zero, but the amplitude of the $m=1$ distorsion tends to take up the incidence angle of the wind. We conclude that this is a very clear case in which the $m=1$ distortion amplitude is much bigger than the $m=0$ amplitude and, therefore, it is possible to have a $m=1$ deformation larger than the $m=0$ deformation.

\subsection{Direction of the warp}

One relevant aspect of the present hypothesis is that it allows us to relate the direction of the warp to the direction of the inflow, $\boldsymbol{v}_{\mathbf{0}}$, with respect to the centre of the galaxy. 


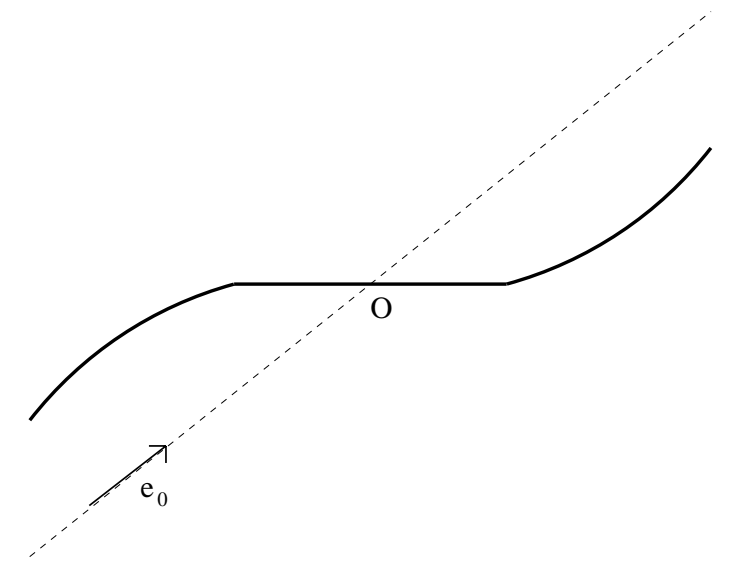

Fig. 9. Graphical representation of the orientation of the warp with respect to the velocity of incoming flow. The azimuthal angle of the warp, which is not plotted, is the same as the azimuthal angle of $\boldsymbol{v}_{\mathbf{0}}$, i.e. $\phi_{0}$.

The torque between two rings, $\mathrm{d} \tau_{S}(R) \mathrm{d} R \mathrm{~d} S$, is proportional to $\left(\sin \phi_{0} \boldsymbol{i}-\cos \phi_{0} \boldsymbol{j}\right)$. This can be demonstrated by use of the Eq. (5), or by a simple analogy with the gravitational torque of one particle (Sect. 2.2): the second ring is the equivalent to an integration of the particle position over the second ring azimuthal angle. Likewise, the external torque is proportional to $\left(\sin \phi_{0} \boldsymbol{i}-\cos \phi_{0} \boldsymbol{j}\right)$. This means that the warp direction is the same of the projection of $\boldsymbol{v}_{\mathbf{0}}$ over the disc, and the precession is around the axis parallel to $\boldsymbol{v}_{\mathbf{0}}$.

The amplitude of the collisional torque, (39), is larger for larger $R$, since $b^{2} \frac{\partial b}{\partial R}$ increases with $R$, so the outer rings have an excess angular velocity of precession compared to the average. However, the outer rings receive a torque from the inner rings, which is proportional to the collisional torque when $\phi_{P}=\phi_{0}$, where $\boldsymbol{e}_{\boldsymbol{P}}$ is the position of the maximum height above the plane of both inner and outer rings (i.e. the direction of the warp), but must act in the opposite direction to counteract the above excess. Hence, the outer rings should be oriented towards positive $z$ if $\theta_{0}$ is positive or towards negative $z$ if $\theta_{0}$ is negative.

From the above considerations, we deduce that the warp is oriented in the direction parallel to the projection of $\boldsymbol{e}_{\mathbf{0}}$ in the disc. The azimuthal angles of the maximum and minimum heights $(z)$ of the warp are $\phi_{0}$ and $\phi_{0}+\pi$ respectively if $\theta_{0}>0$. If $\theta_{0}<0$, the maximum height is at azimuth $\phi_{0}+\pi$ while the minimum is at $\phi_{0}$. Figure 9 shows a graphical representation of these orientations in the plane which contains the vector $\boldsymbol{v}_{\mathbf{0}}$ perpendicular to the disc. Note that the orientation of the warp does not change if the velocity is $-\boldsymbol{v}_{\mathbf{0}}$ instead of $\boldsymbol{v}_{\mathbf{0}}$.

\section{Warp in a typical spiral galaxy due to intergalactic accretion flows}

The proposed hypothesis of formation of warps can be compared with our observational knowledge about galaxies and the intergalactic medium. We must bear in mind that the calculations developed in this paper are based on fairly crude approximations. We have considered particle trajectories as hyperbolic, neglecting the gravitational effects of the extended disc, we have considered a collimated infinite beam, constant density of the intergalactic flow, etc. Therefore, we should not expect the theory to reproduce exactly all the fine details of a warp. However, we will show that our model reproduces quite well the observed warps using a standard model of the disc of a typical spiral galaxy (for instance, the Milky Way) and with entirely reasonable parameters for the intergalactic flow. Our restricted goal here is to offer a general model for warps (a warp amplitude $\alpha(R)$ which is close to zero out to some specific $R$, but rises rapidly at higher increasing $R$ ), and to test it using the parameters of a typical spiral galaxy such as the Milky Way.

\subsection{Amplitude of the warp}

The solution of (12) by means of the numerical method explained in Appendix A gives $\alpha(R)$ for the galactic warp. It will depend on the adopted galactic model. Our purpose is to demonstrate that the order of magnitude of the amplitude of the warp is approximately the observed amplitude, so we use only one model. Varying the parameters of the disc will, of course, lead to variable results but these would not be quantitatively very different. The surface density of the disc we adopt is given by (15) and the rotation velocity is given by (16), both corresponding to the Milky Way.

In the linear regime, for a low amplitude of the warp and low velocity $v_{0}$, in the limits (40) and (6), the proportionality followed is $\alpha(R) \propto \omega_{p} \propto \frac{\rho_{\mathrm{b}} M_{\mathrm{gal}}^{3 / 2}}{v_{0}}$, in which the dependence of $\alpha(R)$ on $R$ is not specified. Specifically, for the adopted disc model and $\left|\theta_{0}\right|=45^{\circ}$, we obtain in this linear regime that the angle of the warp is $\left(\Omega_{b}=0.02 \mathrm{~h}^{-2}\right.$; Schramm \& Turner 1998)

$\alpha\left(2 R_{0}\right) \approx 5.2 \times 10^{-34} \frac{\rho_{\mathrm{b}}\left(\mathrm{kg} / \mathrm{m}^{3}\right) M_{\mathrm{gal}}(\mathrm{kg})^{3 / 2}}{v_{0}(\mathrm{~m} / \mathrm{s})} \mathrm{rad}$

$=1.7 \times 10^{-4} \frac{\rho_{\mathrm{b}}}{\Omega_{\mathrm{b}} \rho_{\text {crit }}}\left(\frac{M_{\mathrm{gal}}}{10^{11} M_{\odot}}\right)^{3 / 2} \frac{100 \mathrm{~km} \mathrm{~s}^{-1}}{v_{0}} \mathrm{rad}$,

and the precession angular velocity

$$
\begin{array}{r}
\omega_{p} \approx 7.7 \times 10^{-21} \frac{\rho_{\mathrm{b}}}{\Omega_{\mathrm{b}} \rho_{\text {crit }}}\left(\frac{M_{\mathrm{gal}}}{10^{11} M_{\odot}}\right)^{3 / 2} \\
\times \frac{100 \mathrm{~km} \mathrm{~s}^{-1}}{v_{0}} \mathrm{rad} / \mathrm{s} .
\end{array}
$$

Burton (1988) gives an observational value of $\alpha\left(2 R_{0}\right) \approx$ $0.14 \mathrm{rad}$ in our Galaxy which, if we take the value at $2 R_{0}$ to normalize the amplitude of the warp, leads to

$$
\rho_{\mathrm{b}} \approx 820 \Omega_{\mathrm{b}} \rho_{\text {crit }}\left(\frac{v_{0}}{100 \mathrm{~km} \mathrm{~s}^{-1}}\right)\left(\frac{M_{\mathrm{gal}}}{10^{11} M_{\odot}}\right)^{-3 / 2}
$$

$\omega_{p} \approx 6.2 \times 10^{-18} \mathrm{rad} / \mathrm{s}=1$ cycle in $32 \mathrm{Gyr}$. 
The precession is far too slow to be significant, and much slower than the rotational velocity of the Galaxy.

We adopt a total mass of the Galaxy of $M_{\text {gal }}=$ $2 \times 10^{11} M_{\odot}$ (Honma \& Sofue 1996) to calculate the curvature of the hyperbolic trajectories. This estimate includes bulge, disc and spheroidal component masses. Hence,

$\rho_{\mathrm{b}} \approx 290 \Omega_{\mathrm{b}} \rho_{\text {crit }}\left(\frac{v_{0}}{100 \mathrm{~km} \mathrm{~s}^{-1}}\right)$.

The only free parameters with observational uncertainties are the mean density of the intergalactic inflow and its velocity which are related by the last equation. Assuming a typical velocity, in galactocentric coordinates, of $v_{0} \sim$ $100 \mathrm{~km} \mathrm{~s}^{-1}$ (the limit of low velocity adopted in (40) is valid if $R$ is much less than 300 or $400 \mathrm{kpc}$, which is certainly the case since we take $R \leq 16 \mathrm{kpc}$ ), we find:

$\rho_{\mathrm{b}} \sim 290 \Omega_{\mathrm{b}} \rho_{\text {crit }}=1.1 \times 10^{-25} \mathrm{~kg} / \mathrm{m}^{3}$,

which is equivalent to

$n_{\mathrm{HI}} \sim 6 \times 10^{-5}{\mathrm{f} \mathrm{cm}^{-3},}^{-3}$

where $f$ is the fraction of HI in the total baryonic mass of the wind. This value coincides with the most probable value estimated by Kahn \& Woltjer (1959) for the mean density of intergalactic matter yielding dynamical stability for the Local Group of galaxies. López-Corredoira et al. (1999) also calculated a total intergalactic mass in the Local Group around $2 \times 10^{12} M_{\odot}$, which in a volume of $\sim 1 \mathrm{Mpc}^{3}$ gives a mean density around $10^{-25} \mathrm{~kg} / \mathrm{m}^{3}$.

The shape of $\alpha(R) \approx|z| / R$ for these values would be that given in Fig. 10. The prediction of this model agrees with many of the features of the observed warp (Burton $1988,1992)$ : it predicts a flat disc which is not significantly warped for values of $R$ less than $\sim 1.3 R_{0}=10 \mathrm{kpc}$, and a warp whose amplitude increases rapidly at larger radii. The shape of the warp depends on the detailed radial variation of $\sigma(R)$ and we have used a rough exponential estimate of this dependence, which is really more complex than a simple exponential function with a constant scale length. In any case, it is not at this stage worth using a more realistic model since our approach is only an approximation, given the simplifications we have employed in the analytical calculation of the torque. The functional shape of $\alpha(R)$ resembles that of the observational data, within the values of $R$ less than $\sim 16 \mathrm{kpc}$ from the centre. Nonlinear effects have a significant influence at larger radii as well as departures from the simple ring model of the disc. Nevertheless, we should note that the dependence of $\boldsymbol{\tau}_{\text {ext }}$ on $R$ does not affect too much the form of $\alpha(R)$ and other external torques may also produce similar shapes, as we have seen in Figs. 3 and 4.

The maximum distortion of the disc due to the transfer of linear momentum (Sect. 3.6) is calculated using the expression (49). The results are also plotted in Fig. 10. In this case, $z$ is always positive or always negative, a cup shape rather than an integral sign shape. From the numbers plotted in this figure, it can be concluded that this distortion is small compared to that produced by the

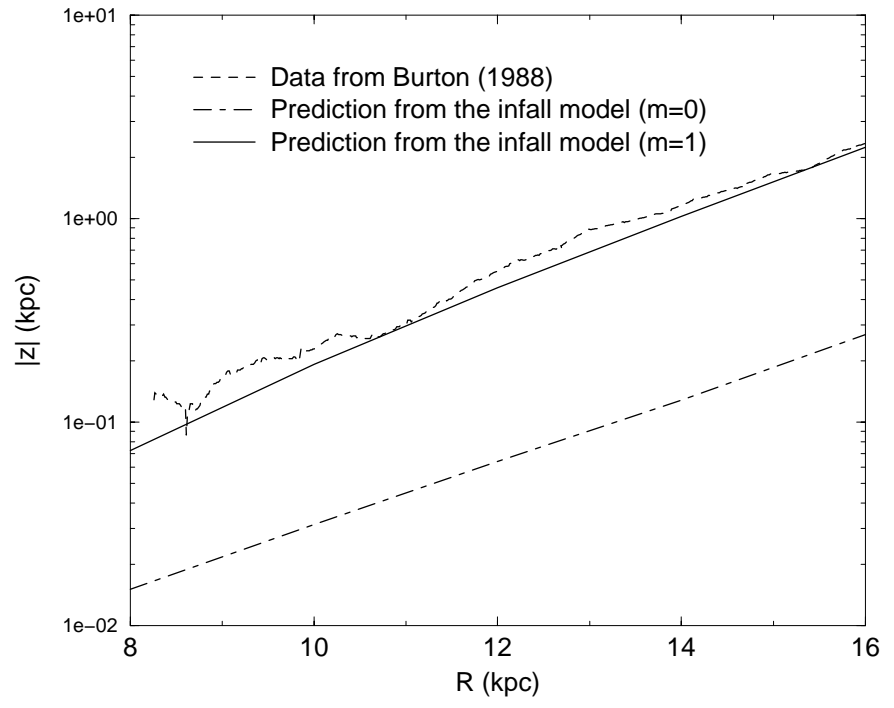

Fig. 10. Milky Way warp maximum height as a function of the radius in the hypothesis of continous accretion of intergalactic matter with $\rho_{\mathrm{b}}=1.1 \times 10^{-25} \mathrm{~kg} / \mathrm{m}^{3},\left|\theta_{0}\right|=45^{\circ}(\mathrm{m}=1$, solid line). For comparison, a maximum limit of the effects of the linear momentum increment over the rings are shown $(m=0$, dot-dashed line). Dashed line stands for observational data of the northern warp (Burton 1988) rescaled to $R_{0}=7.9 \mathrm{kpc}$.

torque, i.e. the integral sign shape will be predominant in the galactic disc. We think the predominance of torque effects is due to the smaller mass concentrated in the disc in comparison to the total mass of the Galaxy, which is mostly responsible of the counter-torques which reduce the heightness of the warp $m=1$, while the whole mass of the Galaxy produces the counter-forces which reduce the distortion $m=0$. In some galaxies, a mixture of cupshape and integral sign shape could be produced by this mechanism. This would be a possible explanation for the asymmetries between the two sides of the warp, even for our own Galaxy. It will depend, among other factors, on the angle $\theta_{0}$. For $\theta_{0}=90^{\circ}$, the transfer of vertical linear momentum would be maximum while the torque would be zero. Therefore, there is a probability, although small, of finding cup-shaped distortions of galactic discs rather than integral-sign warps. In Fig. 11, we show that the cupshaped distortion is predominant for $\left|\theta_{0}\right|>\sim 85^{\circ}$, which translates to a probability that a cup-shape is dominant of $<\sim 4 \times 10^{-3}$, for spiral galaxies like the Milky Way. It would be of interest to make a statistical search over a major sample of warped galaxies to see how many galaxies present appreciable cup-shaped distortion. The study of the fraction of galaxies which present irregulatities within the integral sign warp would also give some clue about the validity of this hypothesis.

Near the limit $\theta_{0}=0$ the amplitude of the warp goes to zero quickly. Although, Fig. 11 shows apparently a vertical tangent in the limit $\theta_{0}=0$, that is not the case: there is a horizontal tangent, as the limit of (39) when $\theta_{0}$ is very small is proportional to $\theta_{0}^{2}$. However, this limit manifests for very small angles and this is the reason for the apparent 


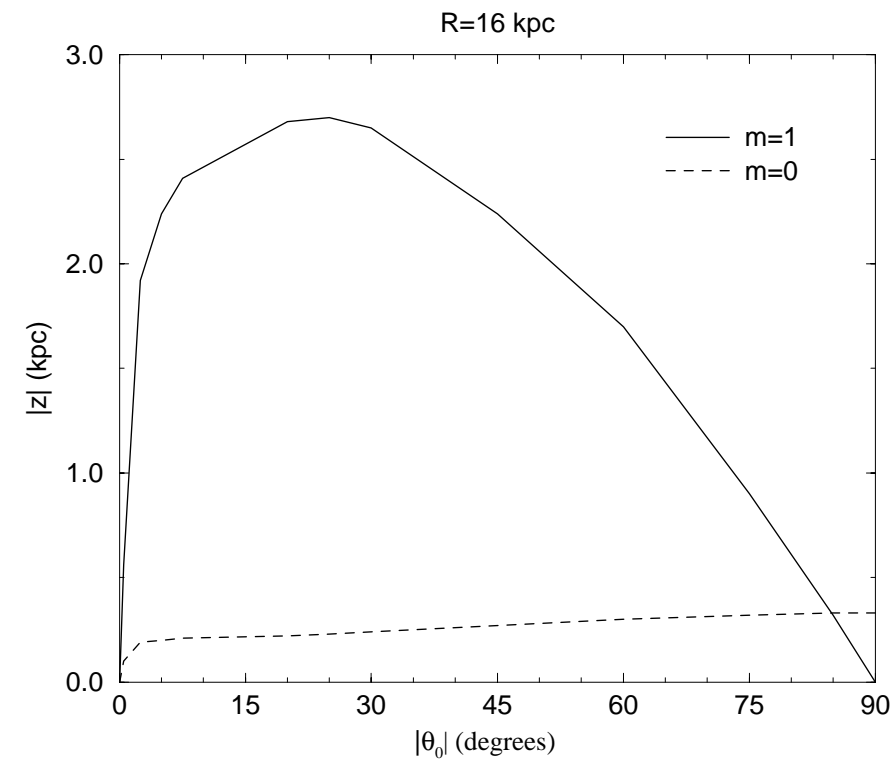

Fig. 11. Milky Way warp maximum height as a function of the inclination of the incoming flow with respect to the galactic plane $\left(\theta_{0}\right)$ in the hypothesis of continous accretion of intergalactic matter: $m=1$ (integral-sign warp due to a torque), solid line; $m=0$ (cup-shaped distortion due to a force), dashed line.

vertical tangent in 11 . Indeed, the values are: $|z|\left(\theta_{0}=\right.$ $\left.10^{\circ}\right)=2.45 \mathrm{kpc},|z|\left(\theta_{0}=5^{\circ}\right)=2.24 \mathrm{kpc},|z|\left(\theta_{0}=2^{\circ}\right)=$ $1.79 \mathrm{kpc},|z|\left(\theta_{0}=1^{\circ}\right)=1.24 \mathrm{kpc},|z|\left(\theta_{0}=0.5^{\circ}\right)=0.59$ $\mathrm{kpc},|z|\left(\theta_{0}=0.25^{\circ}\right)=0.19 \mathrm{kpc},|z|\left(\theta_{0}=0.1^{\circ}\right)=0.034$ $\mathrm{kpc}, \ldots, z\left(\theta_{0}=0\right)=0 \mathrm{kpc}$.

We can add some further comments about the stellar warp. In the Milky Way, the OB stars follow the gas (Porcel \& Battaner 1995). But it is not just the young population, recently formed from the warped gas, which shows a stellar warp, but the whole population of stars (Porcel et al. 1997; Dehnen 1998). The whole population of stars of the Milky Way projected onto the sky appears less deviated from the plane than the projected gas, but this does not necessarily represent a difference between amplitudes of the gaseous and stellar warps. Rather, it may well be due to the cut-off of the stellar population at a smaller Galactocentric radius than the gas, around $15 \mathrm{kpc}$ (Porcel et al. 1997). The stellar disc is clearly warped, perhaps somewhat less than the gaseous disc although not obviously so. As pointed out in Sect. 3.3, if the stellar disc were demonstrated to be less warped than the gas warp, it would be evidence supporting either this theory or the theory of the intergalactic magnetic field as the generator of the warp (Porcel et al. 1997). In fact, this would be predicted by a theory in which the external torque directly affects the gas disc and only indirectly the stellar disc.

\subsection{Possible scenarios}

Possible scenarios for the intergalactic flow postulated here are:

1. The galaxy is passing through a continuous intergalactic medium, i.e. the velocity of the flow is due to the relative motion of the galaxy with respect to the rest frame of the intergalactic medium. In this case, the flow may be well approximated by a beam of infinite extent. This may be a representative scenario for most galaxies, and could explain why most spiral galaxies are warped. Asymmetries in the warp could also be explained in terms of a transfer of both linear and angular momentum, although the cases when cup-shaped distortions dominate has low probability. We think that this first item is the most plausible explanation.

2. The galaxy has an interacting companion and some exchange of material is produced due to tidal effects. The stream of material goes from the companion to the main galaxy, and this flow could also generate a warp. The direction of the wind would not be constant, because the companion is orbiting around the main galaxy so the warp would not be steady. This scenario would give asymmetries in the warp, apart from that coming from the $m=0$ component, which would be due to the non-symmetric form of the infalling material, with finite impact parameter, or clouds which intersect the centre of the galaxy in a non-axisymmetric way. Reshetnikov \& Combes (1998) find some correlation between the frequency of warps and the interaction with other galaxies. An accretion inflow due to the exchange of material with a companion might appear to be undermined by the observations of warped galaxies which are apparently isolated. However, this phenomenon is itself being challenged by the discovery of some companions to galaxies which had been considered as classical examples of isolated galaxies (for instance, NGC 5907; Shang et al. 1998).

While the present paper was in the refereeing process, a work by García-Ruiz (2001) has been published. This is an interesting work which analyzes in detail 26 edge-on galaxies in radio and optical. García-Ruiz (2001) finds that 20 galaxies are warped, two of them present U-warp, and 7 with a warp of only one side. These asymmetric cases can be explained by the present theory as a combination of $m=0$ and $m=1$ distortion and it is interesting to note that up to now there is no alternative explanation for the U-warps as well as the asymmetric warps. He also found that the frequency of warps and its amplitude is dependent on environment. It is even more interesting to note that the most isolated galaxies are more frequently warped (although with less amplitude). "While this suggests that tidal interaction plays a role in warping, it seems likely that there are other effects at work that cause even quite isolated galaxies to warp." (García-Ruiz 2001). It seems clear that warping is due to something related with the environment rather that the intrinsic properties of the galaxies, and something which is not related with the proximity of other galaxies. The accretion of intergalactic matter onto the disc seems a good candidate to explain these observational facts.

The density of the intergalactic medium is very low on average, making it difficult to detect. However, it could 
well be that the High Velocity Clouds (HVCs; see the reviews in Wakker \& van Woerden 1997; Wakker et al. $1999 \mathrm{~b})$ are part or all of this material falling towards the Galactic disc from all directions. If these do produce a net torque it must be because there is a net galactocentric average velocity of the complete set of HVCs, including the Magellanic Stream, the complexes and the HVCs associated with more distant clouds infalling towards the Local Group barycenter (Blitz et al. 1999; Braun \& Burton 1999; López-Corredoira et al. 1999). The tidal Stream from the Saggitarius dwarf galaxy (Ibata et al. 2001) might also take part. An average hydrogen density for the flux of $n_{\mathrm{HI}} \sim 6 \times 10^{-5} \mathrm{f} \mathrm{cm}^{-3}$ is in good agreement with the average density of an $\mathrm{HVC},\left\langle n_{\mathrm{HI}}\right\rangle \sim 10^{-4}-10^{-1} \mathrm{~cm}^{-3}$ (Blitz et al. 1999; Wakker et al. 1999a), including the value of $f$ which may be as low as 0.02 (Braun \& Burton 2000) if the matter of the flow is baryonic. The mean density in the intergalactic clouds may be enough to create the Galactic warp. If the density of infalling matter were equal to that of an individual HVC, we would have a higher density than that required, by several orders of magnitude. However, we know that the intracluster medium is not filled by HVCs; these represent a low volume high density fraction; averaging over the complete medium with plausible filling factor can yield a net hydrogen density around $n_{\mathrm{HI}} \sim 6 \times 10^{-5} \mathrm{f} \mathrm{cm}^{-3}$.

The degree of clumpiness of the intergalactic medium is not well known. However, it is not of importance whether the flow is continuous or discretized in clouds. The warp will be produced by the average infall. Shortterm fluctuations of the infalling density do not appreciably affect the warp since the forces responsible for it have a very low amplitude and require a long time to produce or distort the warp.

Using expression (41), we can derive a total accretion rate of the Galactic disc out to $R_{\max }=15 \mathrm{kpc}$ of $\sim 1 M_{\odot} /$ yr for this density. This turns out to be of the order of the accretion rate required to resolve the G-dwarf problem in our Galaxy as well as explaining a number of phenomena of chemical evolution which require the longterm infall of low metallicity gas (López-Corredoira et al. 1999; Wakker et al. 1999a). The infall of 1 solar mass per year is enough to produce the warp because: 1) the external disc has a very low density and small forces produce considerable accelerations; 2 ) the acceleration may in fact be very small in amplitude, but the period of time to produce the warp is large enough (order of Gyr), so this gives time to distort the galactic disc (in $1 \mathrm{Gyr}, 10^{9}$ solar masses are accreted which is a significant quantity of accreted mass).

This hypothesis could, in a general way, explain the possible alignments of the different warps of neighbouring galaxies (Battaner et al. 1991) if the flow velocity is similar around such galaxies within the same zone of the intergalactic medium.

Whatever the structure and composition of the intergalactic medium, it is clear that intergalactic space is by no means empty, and the accretion of this material by galaxies is likely to have been continuous during their lifetimes. The effects of this accretion can be detected in their chemical evolution as well as in their structure, as pointed out above.

\section{The effects of a very massive halo}

Although we already discussed the effects of the halo, we are going to clarify the use of the halo here as well as the effects which a very massive halo could produce.

We may infer from the present calculations that we have found a mechanism which could explain both qualitatively and quantitatively the generation of warps in normal spiral galaxies, and this mechanism is the interaction between the disc and the infalling matter as well as the interaction of the disc with itself (rings interacting with other rings). The dark halo is included although it is not explicit in some calculations of this paper, but its effect is not very important for a rough calculation in which we are interested in the order of magnitude. In fact, our calculations are not rough but almost exact, but the roughness of the numbers obtained is due the uncertainties in the different parameters.

Briefly, these are the effects which are treated here, and others which have not been treated but require treatment in future papers:

- The dark halo is present in the total mass of the Galaxy: $M_{\text {gal }}$. The assumed value of the Milky Way mass of $2 \times 10^{11} M_{\odot}$ within a radius $R \sim 20 \mathrm{kpc}$ from the centre includes the halo, some of whose matter is not visible. The effects of the halo are also implicitly included in the calculation of $M_{\text {gal }}(R)$ as a function of $v_{\text {rot }}(R)$ from the rotation curves.

- The interaction of the halo with the warp is not considered, but we have made estimates to show that for this case the effect is small compared to the countertorque of the disc. Therefore, we feel that the exclusion of halo effects in the countertorques is a fair approximation if we are interested only to find the order of magnitude of the warp amplitude. The countertorques of the halo are less important than those of the disc because the halo is much closer to sphericity. We showed in Sect. 2 that the contribution of the halo countertorque for $R<16 \mathrm{kpc}$ is less than $\sim 40 \%$ of the disc contribution. The uncertainties in the halo mass distribution lead to an uncertainty similar to this value and we did not feel that much would be gained by adding the extra complexity. We know that the warp would be reduced by this effect, but this reduction is not the dominant term.

- A very massive and very extended halo would introduce some variations in the numbers we derived although the mechanism would work qualitatively in a similar way. First, if it is very massive $M_{\text {gal }}$ would be larger and the amplitude of the warp would be correspondingly larger. We have shown that the amplitude of the warp is proportional to $M_{\text {gal }}^{3 / 2}$. This, however, 
was on the assumption that most of the mass is concentrated within a radius less than $\sim 20 \mathrm{kpc}$. If the halo is very extended, this approximation is not appropriate. We should then need to consider the mass distribution of the halo instead of assuming a point mass to calculate the infall velocity of the clouds. The problem would be much more complex because we would need a gravitational potential different from $\phi \propto 1 / r$ and the trajectories would not be hyperbolae. Indeed, the mechanism we propose to form warps works with any potential, but the calculations are of course much easier for a $\phi \propto 1 / r$ law. Since we are interested in proposing a new mechanism and showing how it works, we think that these complexities should be left for a future paper. At present, we can say that a very massive halo would increase the amplitude of the warp, not proportionally to $M_{\text {gal }}^{3 / 2}$ but some other power with exponent less than $3 / 2$. This is because the infall velocity of the clouds is increased as well as the curvature of the trajectories. It is important to note that a very extended halo would not differ from a halo constrained within $R<20 \mathrm{kpc}$ in the countertorques produced on the warp because only the mass in the ellipsoids internal to the radius of the warp produce gravitational torque, assuming there is a constant ellipticity halo. To summarize, with a very massive halo our mechanism does operate, even in fact more effectively because it would require a lower intergalactic density to produce the same warp amplitude.

- If the halo axis were displaced with respect the disc axis the disc would be pinched by the halo. This was the case studied by Ostriker \& Binney (1989), Binney et al. (1998) or Jiang \& Binney (1999) and is indeed a mechanism which will give rise to warps too. The scenario presented by these authors is different from the scenario proposed here. They assume the infall of the intergalactic matter onto the halo rather than the disc, and this produces motion of the halo with respect to the disc. We do not seek to challenge this in the present paper, rather to present a possible alternative, which might be complementary. The compatibility of the two mechanisms certainly merits effort to study further. There is no doubt that accretion into a halo which gives an offset in the rotation axis from the disc axis will give rise to a warp; if one accepted the possibility of a low density halo accreting intergalactic matter, one should recognize the possibility of giving rise to warps by this mechanism. We have shown here how accretion directly onto the disc can also yield a warp, with parameters in the observed range. At the present stage of understanding the problem, we think that either or both mechanisms can act to produce warps if one accepted that the accretion of matter by the disc or the halo have the same plausibility. Our opinion is that our mechanism is preferable since the accretion of matter by the disc is more plausible than the accretion by the halo but this point is open for discusion.

\section{Conclusions}

We propose that galactic warps are produced by the reorientation of the galactic disc structure in order to compensate the differential precession due to a torque generated by an external force. The external force might be the gravitational interaction with a satellite, but in the case of the Milky Way, with the Magellanic Clouds as the satellites, there is not enough mass close enough to provide the observed amplitude in the warp. Magnetic forces could also produce the warp, but the intergalactic field would then be of the order of $\mu \mathrm{G}$.

A simple model of an intergalactic accretion flow which intersects a galactic disc (or, equivalently, considering the galaxy as moving through the intergalactic medium) can explain the existence of warps in the galaxy if the mean density of baryonic matter in the medium is around $10^{-25} \mathrm{~kg} / \mathrm{m}^{3}$ and the infall velocity at large distance is $\sim 100 \mathrm{~km} \mathrm{~s}^{-1}$. This hypothetical low density flow is a very reasonable physical assumption and would explain why most spiral galaxies are warped.

Accretion due to such a flow is in good accord with the observations of the chemical evolution of the Milky Way by contributing $\sim 1 M_{\odot} /$ yr of low metallicity gas to the disc. The High Velocity Clouds, which are presumably the accretable material in the Local Group galaxies (Blitz et al. 1999; Braun \& Burton 1999; López-Corredoira et al. 1999; Wakker et al. 1999a) are candidates for a significant fraction of the material which fills intergalactic space and is accreted. No massive halo is necessary nor high values of magnetic fields are necessary, although the presence of these elements would not modify qualitatively the present conclusions. No calculations in the framework of accretion onto the disc are given for a very massive halo but its effect would not affect qualitatively the present mechanism, as it is explained in Sect. 5. Models with a very massive halo could be generated and different numerical results would be obtained depending on the parameters of the halo, although no major qualitative changes are expected to the model presented here since the mechanism of formation of warps is dominated by the interaction with the disc; only quantitative changes would come from the increasing velocity and trajectory curvature of the infalling material which would increase the amplitude of the warp, i.e. the same amplitude of the warp would be obtained with a density even lower than $10^{-25} \mathrm{~kg} / \mathrm{m}^{3}$.

Several mechanisms can generate warps but, among them, accretion of an intergalactic flow seems to offer a very plausible scenario: it is quantitatively consistent with many observations and works independently of other ingredients of galaxies and their structure.

Acknowledgements. We particularly appreciate the comments of the referees - E. Battaner and the anonymous referee - and of J. J. Binney and I. Shlosman, whose detailed questions have helped us to explain more clearly some important technical points. This work has been supported by grant PB97-0219 of the Spanish DGES. 


\section{Appendix A: Numerical calculation of $\alpha(R)$}

The solution of $\frac{\mathrm{d} \omega_{p}(R)}{\mathrm{d} R}[\alpha(R)]=0$, from (12), can be achieved by means of a numerical calculation with $N$ discreet values of $R$, i.e.

$\boldsymbol{H}(\boldsymbol{\alpha})=\mathbf{0}$,

$\boldsymbol{\alpha}=\left(\alpha_{1}, \ldots, \alpha_{N}\right) ; \boldsymbol{H}=\left(H_{1}, \ldots, H_{N}\right)$,

$\alpha_{i} \equiv \alpha\left(R_{i}\right) ; H_{i} \equiv \frac{\mathrm{d} \omega_{p}}{\mathrm{~d} R}\left(R_{i}\right)$

Newton-Raphson's iterative method is appropriate for this kind of numerical calculations. The iteration $k+1$ is given by

$\boldsymbol{\alpha}^{k+1}=\boldsymbol{\alpha}^{k}-\mathcal{W}^{-1}\left(\boldsymbol{\alpha}^{k}\right) \boldsymbol{H}\left(\boldsymbol{\alpha}^{k}\right)$

$\mathcal{W}_{=}\left(\begin{array}{lll}W_{11} & \ldots & W_{1 N} \\ \ldots & \ldots & \ldots \\ W_{N 1} & \ldots & W_{N N}\end{array}\right) \quad ; W_{i j}=\frac{\partial H_{i}}{\partial \alpha_{j}}$

By means of this application, we can calculate $\alpha(R)$. The first iteration is taken with $\alpha(R)=\left|\theta_{0}\right|$.

\section{References}

Bahcall, J. N., \& Soneira, R. M. 1980, ApJS, 44, 73

Battaner, E., Florido, E., \& Sánchez-Saavedra, M. L. 1990, A\&A, 236, 1

Battaner, E., Garrido, J. L., Membrado, M., \& Florido, E. 1992, Nature, 360, 652

Battaner, E., Garrido, J. L., Sánchez-Saavedra, M. L., \& Florido, E. 1991, A\&A, 251, 402

Battaner, E., \& Jiménez-Vicente 1998, A\&A, 332, 809

Binney, J. J. 1991, in Dynamics of Disc Galaxies, ed. B. Sundelius, Chalmers Univ., Göteborg: Dep. Astronomy, 297

Binney, J. J. 1992, ARA\&A, 30, 51

Binney, J. J. 2000, in Dynamics of Galaxies: from the Early Universe to the Present, ASP Conf. Ser., 197, ed. F. Combes, G. A. Mamon, \& V. Charmandaris (ASP, San Francisco), 107

Binney, J. J., Jiang, I.-G., \& Dutta, S. N. 1998, MNRAS, 297, 1237

Binney, J. J., \& May, A. 1986, MNRAS, 218, 743

Blitz, L., Spergel, D., Teuben, P., Hartmann, D., \& Burton, W. B. 1999, ApJ, 514, 818

Braun, R., \& Burton, W. B. 1999, A\&A, 341, 437

Braun, R., \& Burton, W. B. 2000, A\&A, 354, 853

Briggs, F. H. 1990, ApJ, 352, 15

Burton, W. B. 1988, in Galactic and Extragalactic Radio Astronomy, ed. K. I. Kellerman, \& G. L. Verschuur (Springer-Verlag, Berlin), 295

Burton, W. B. 1992, in The Galactic Interstellar Medium, ed. D. Pfenniger, \& P. Bartholdi (Springer-Verlag, Berlin), 126
Casuso, E., \& Beckman, J. E. 1997, ApJ, 475, 155

Casuso, E., \& Beckman, J. E. 2000, PASP, 112, 942

Debattista, V., \& Sellwood, J. 1999, ApJ, 513, L107

Dehnen, W. 1998, AJ, 115, 2384

Evans, N. W. 2001, in IDM 2000: Third International Workshop on the Identification of Dark Matter, ed. N. Spooner (World Scientific, Singapore), in press [astro-ph/0102082]

García-Ruiz, I., Kuijken, K., \& Dubinski, J. 2000, MNRAS, submitted [astro-ph/0002057]

García-Ruiz, I 2001, Ph.D. Thesis, Univ. Groningen (Holland)

Honma, M., \& Sofue, Y. 1996, PASJ, 48, L103

Hunter, C., \& Toomre, A. 1969, ApJ, 155, 747

Ibata, R. A., Irwin, M., Lewis, G., \& Stolte, A. 2001, ApJ, 547, $\mathrm{L} 133$

Ibata, R. A., \& Razoumov, A. O. 1998, A\&A, 336, 130

Ideta, M., Hozumi, S., Tsuchiya, T., \& Tazikawa, M. 2000, MNRAS, 311, 733

Jiang, I.-G., \& Binney, J. 1999, MNRAS, 303, L7

Kahn, F. D., \& Woltjer, L. 1959, ApJ, 130, 705

Kronberg, P. P. 1994, Rep. Progress in Phys., 57, 325

Kuijken, K., \& Dubinski, J. 1995, MNRAS, 277, 1341

Kuijken, K., \& Gilmore, G. 1989, MNRAS, 239, 605

Lin, D. N. C., \& Lynden-Bell, D. 1982, MNRAS, 198, 707

López-Corredoira, M., Beckman, J. E., \& Casuso, E. 1999, A\&A, 351, 920

López-Corredoira, M., Hammersley, P. L., Garzón, F., Simonneau, E., \& Mahoney, T. J. 2000, MNRAS, 313, 392

Lynden-Bell, D. 1965, MNRAS, 129, 299

Murai, T., \& Fujimoto, M. 1980, PASJ, 32, 581

Nelson, A. H. 1988, MNRAS, 233, 115

Nelson, R. W., \& Tremaine, S. 1995, MNRAS, 275, 897

Ostriker, E. C., \& Binney, J. J. 1989, MNRAS, 237, 785

Porcel, C., \& Battaner, E. 1995, MNRAS, 274, 1153

Porcel, C., Battaner, E., \& Jiménez-Vicente, J. 1997, A\&A, 322, 103

Reshetnikov, V., \& Combes, F. 1998, A\&A, 337, 9

Rocha-Pinto, H. J., Maciel, W. J., Scalo, J., \& Flynn, C. 2000, A\&A, 358, 850

Rogstad, D. H., Lockhard, I. A., \& Wright, M. C. H. 1974, ApJ, 193, 309

Ryden, B. S. 1988, ApJ, 329, 589

Ryden, B. S., \& Gunn, J. E. 1987, ApJ, 318, 15

Sánchez-Saavedra, M. L., Battaner, E., \& Florido, E. 1990, MNRAS, 246, 458

Schramm, D. N., \& Turner, M. S. 1998, Rev. Mod. Phys., 70, 303

Shang, Z., Brinks, E., Zheng, Z., et al. 1998, ApJ, 504, L23

Tinsley, B. M. 1980, Fund. Cosmic Phys., 5, 287

Wakker, B. P., \& van Woerden, H. 1997, ARA\&A, 35, 217

Wakker, B. P., Howk, J. C., Savage, B. D., et al. 1999a, Nature, 402, 308

Wakker, B. P., van Woerden, H., \& Gibson, B. K. 1999b, in Stromlo Workshop on High-Velocity Clouds, ed. B. K. Gibson, \& M. E. Putman, ASP Conf. Ser., 166, 311

Weinberg, M. D. 1998, MNRAS, 299, 499 